\title{
Partition function of free conformal fields in 3-plet representation
}

\author{
Matteo Beccaria ${ }^{a}$ and Arkady A. Tseytlin ${ }^{b, 1}$ \\ ${ }^{a}$ Dipartimento di Matematica e Fisica "Ennio De Giorgi", Università del Salento Es INFN, \\ Via Arnesano, 73100 Lecce, Italy \\ ${ }^{b}$ The Blackett Laboratory, Imperial College, \\ London $S W^{r}$ 2AZ, U.K. \\ E-mail: matteo.beccaria@le.infn.it, tseytlin@imperial.ac.uk
}

AbSTRACT: Simplest examples of AdS/CFT duality correspond to free CFTs in $d$ dimensions with fields in vector or adjoint representation of an internal symmetry group dual in the large $N$ limit to a theory of massless or massless plus massive higher spins in $\operatorname{AdS}_{d+1}$. One may also study generalizations when conformal fields belong to higher dimensional representations, i.e. carry more than two internal symmetry indices. Here we consider the case of the 3-fundamental ("3-plet") representation. One motivation is a conjectured connection to multiple M5-brane theory: heuristic arguments suggest that it may be related to an (interacting) CFT of $6 \mathrm{~d}(2,0)$ tensor multiplets in 3-plet representation of large $N$ symmetry group that has an $\mathrm{AdS}_{7}$ dual. We compute the singlet partition function $Z$ on $S^{1} \times S^{d-1}$ for a free field in 3-plet representation of $\mathrm{U}(N)$ and analyse its novel large $N$ behaviour. The large $N$ limit of the low temperature expansion of $Z$ which is convergent in the vector and adjoint cases here is only asymptotic, reflecting the much faster growth of the number of singlet operators with dimension, indicating a phase transition at very low temperature. Indeed, while the critical temperatures in the vector $\left(T_{c} \sim N^{\gamma}, \gamma>0\right)$ and adjoint $\left(T_{c} \sim 1\right)$ cases are finite, we find that in the 3 -plet case $T_{c} \sim(\log N)^{-1}$, i.e. it approaches zero at large $N$. We discuss some details of large $N$ solution for the eigenvalue distribution. Similar conclusions apply to higher $p$-plet representations of $\mathrm{U}(N)$ or $O(N)$ and also to the free $p$-tensor theories invariant under $[\mathrm{U}(N)]^{p}$ or $[O(N)]^{p}$ with $p \geq 3$.

Keywords: AdS-CFT Correspondence, Conformal Field Theory

ARXIV EPRINT: 1703.04460

\footnotetext{
${ }^{1}$ Also at Lebedev Institute, Moscow, Russia.
} 


\section{Contents}

1 Introduction $\quad \mathbf{2}$

1.1 Heuristic motivation: 3-plet (2,0) tensor multiplet as M5-brane theory 3

1.2 Structure of the paper 5

2 Partition function with singlet constraint $\quad 6$

$3 \quad N=\infty$ limit of low temperature expansion of $\mathrm{U}(N)$ partition function 9

3.1 Vector and adjoint cases $\quad 9$

3.2 3-plet case: low temperature expansion of $Z$ and counting of operators $\quad 10$

3.3 Exact expression for low temperature expansion of 3-plet partition function in the $N \rightarrow \infty$ limit $\quad 13$

4 Large $N$ partition function and phase transitions $\quad 16$

$\begin{array}{lll}4.1 \text { Overview } & 16\end{array}$

$\begin{array}{lll}4.2 & \text { Large } N \text { limit in terms of eigenvalue density } & 17\end{array}$

$\begin{array}{lll}4.3 & \text { Vector and adjoint cases } & 19\end{array}$

4.4 3-plet case 20

4.4.1 Numerical analysis 20

4.4.2 Exact solution for the eigenvalue density at large $N \quad 21$

5 Concluding remarks $\quad 24$

A $N=\infty$ partition function for 2-plet representation of $\mathrm{U}(N) \quad 25$

B Finite $N$ low temperature expansion of 3-plet partition function $\quad 26$

C Details of analysis of large $N$ partition function for 3-plet representation 27

$\begin{array}{lll}\text { C.1 } & \mathrm{U}(2) \text { case } & 27\end{array}$

C.2 One-harmonic solution: value of the action for the eigenvalue density 28

$\begin{array}{lll}\text { C.3 Including higher harmonics } & 29\end{array}$

C.4 Eigenvalue density for (anti) symmetric 3-plet representation 30

D $N=\infty$ limit of low temperature expansion of $O(N)$ partition function 32

E General expression for single-trace partition function 36

F Singlet partition function of $[\mathrm{U}(N)]^{p}$ invariant $p$-tensor theory $\quad 37$ 


\section{Introduction}

Examples of unitary conformal field theories are free theories containing scalar, spinor or $\left(\frac{d}{2}-1\right)$-form fields in $d$ dimensions. Assuming that these fields transform in some representation of a global $\mathrm{U}(N)$ or $O(N)$ symmetry group one expects that in the large $N$ limit this theory should be dual to a theory in $\mathrm{AdS}_{d+1}$. The latter should contain a tower of massless higher spins dual to bilinear conserved currents as well as an infinite collection of massive higher spins dual to primary operators containing irreducible ("single-trace") contractions of more than two fields.

The simplest case is that of a fundamental (vectorial) representation [1-3] where the spectrum is particularly simple, containing conserved currents in $C F T_{d}$ or massless higher spins in $\mathrm{AdS}_{d+1} .{ }^{1}$ The dual AdS theory is then a massless higher spin theory with $N$ as the coefficient in front of the classical action.

Another well-known example is when the free fields (e.g. spin-1 vectors in $4 \mathrm{~d}$ ) belong to the adjoint representation [5-9]. Here the dual AdS theory should be string-like: in addition to massless higher spin fields it should contain also families of massive fields which together may have an interpretation of a spectrum of a "tensionless" string theory in $\mathrm{AdS}_{d+1} \cdot{ }^{2}$ The coupling constant on the AdS side is then $1 / N$, i.e. the coefficient in front of the AdS field theory action is $N^{2}$.

Here we would like to study the next in complexity case when the CFT field belongs to a 3-fundamental or "3-plet" representation, i.e. to a general, symmetric or antisymmetric 3 -index tensor representation of a global symmetry group. Already in the simplest case of the scalar field the spectrum of "single-trace" operators with more than two fields (dual to massive fields in AdS) here is much more intricate than in the adjoint case, suggestive of a "tensionless membrane" interpretation. The coefficient in front of the dual AdS field theory action should now be $N^{3}$.

The spectrum of CFT operators on $\mathbb{R}^{d}$ (or states in $\mathbb{R} \times S^{d-1}$ ) is naturally encoded in the small temperature $T=\beta^{-1}$ or small $x=e^{-\beta}$ expansion of the partition function $Z(x)$ on $S_{\beta}^{1} \times S^{d-1}$. The singlet-state large $N$ partition function was previously computed in the $\mathrm{U}(N)[10]$ and the $O(N)[11,12]$ vector representation case and was matched to the corresponding massless higher spin partition function in AdS. The partition function in the adjoint representation case was computed in [13-15] (see also [16-18]) and its matching to the AdS partition function was discussed in [7-9]. Once the temperature becomes large enough (of order $N^{\gamma}$ with $\gamma>0$ in the vector case and $\gamma=0$ in the adjoint case) these partition functions exhibit phase transitions $[10,13,15]$ that may have some dual AdS interpretation (cf. [19]).

Here we will compute the singlet partition function $Z(x)$ in the case of a free CFT in a 3 -plet (i.e. 3-index tensor) representation of internal symmetry group. ${ }^{3}$ We shall analyse its

\footnotetext{
${ }^{1}$ In the $\left(\frac{d}{2}-1\right)$-form (e.g. 4 d vector field $)$ case there are also few additional massive fields [3, 4].

${ }^{2}$ In the maximally supersymmetric case this interpretation is of course suggested by the canonical example of duality between the $\mathcal{N}=4$ SYM theory and the superstring theory in $\mathrm{AdS}_{5} \times \mathrm{S}^{5}$ where taking the 't Hooft coupling to zero corresponds to a "tensionless" limit in the quantum string theory.

${ }^{3}$ We shall consider the general (reducible) rank 3 tensor $\mathrm{U}(N)$ representation as well as totally symmetric and antisymmetric cases. We shall also discuss the case of the 3-plet representation of $O(N)$. The large $N$ results will be similar in all of these cases.
} 
small temperature expansion matching the direct operator count and also present the large $N$ solution of the corresponding matrix model implying the presence of a phase transition at small $x_{c}=e^{-\beta_{c}}$ of order of $N^{-a}$ with $a>0$, i.e. at $T_{c}=\frac{a}{\log N}$.

\subsection{Heuristic motivation: 3-plet $(2,0)$ tensor multiplet as M5-brane theory}

Before turning to the details of analysis of the large $N$ limit of the 3 -plet partition function let us first make some speculative remarks providing a motivation behind this work which is related to attempts to understand a $6 \mathrm{~d}$ CFT describing $N \gg 1$ coinciding M5-branes [20$32]^{4}$

The idea that $N^{3}$ scaling of observables in the multiple M5-brane case may be explained in terms of M2-branes with three holes attached to three different M5-branes thus leading to 3 -index world volume fields was originally suggested in [21]. Then a natural proposal that a $6 \mathrm{~d}$ superconformal theory describing multiple M5-branes should involve $(2,0)$ tensor multiplets in 3-tensor representation of $O(N)$ was made in $[27,30] .^{5}$

To recall, the structure of a world-volume theory of a single M5-brane follows from consideration of symmetries and collective coordinates corresponding to the classical M5brane solution in $11 \mathrm{~d}$ supergravity [40-42]. It is represented by a free $(2,0)$ tensor multiplet containing (anti)selfdual 3-form $H_{\mu \nu \lambda}=3 \partial_{[\mu} B_{\nu \lambda]}, 5$ scalars $\phi_{r}$ and 2 Weyl fermions $\psi_{a}$. This is an example of a free $6 \mathrm{~d}$ CFT with conformal anomalies and correlators that can be directly computed [27, 28].

In the case of multiple D-branes the low energy theory is the SYM theory, i.e. one gets $N^{2}$ vector multiplets at weak coupling and that matches the leading $N^{2}$ scaling predicted by the dual supergravity for BPS observables. By formal analogy, one needs $N^{3}$ free tensor multiplets to match the leading large $N$ scaling of protected 11d supergravity observables $[20,21,24,27,28]$. This suggests that the $(2,0)$ multiplet should carry a 3 index representation of an internal $O(N)$ symmetry group that has dimension $\propto N^{3}$ at large $N{ }^{6}$ Thus, if the conjectured $6 \mathrm{~d}$ theory were to have a weak coupling limit its field content would be $\left(B_{\mu \nu}^{i j k}, \phi_{r}^{i j k}, \psi_{a}^{i j k}\right)$ with $i, j, k=1,2, \ldots, N$.

The reason why the M5-brane world-volume 2-form field may carry 3 internal indices can be heuristically argued for as follows [20, 21, 27]. Replacing the standard picture of multiple D-branes connected by open strings by M5-branes connected by M2-branes one may attempt to explain the $N^{3}$ scaling of multiple M5-brane entropy [20] by assuming that

\footnotetext{
${ }^{4}$ Let us note that a possible connection of multiple M5-brane theory to interacting tensor models was mentioned in footnote 1 in [33]. Interacting tensor models [34-36] in large $N$ limit in one dimension where recently investigated in $[33,37]$ in connection with SYK model. Some properties of scalar field tensor models in $d>1$ were studied in [33].

${ }^{5}$ Note that the suggestion to consider tensor multiplets in a 3-plet representation is different from attempts to construct an interacting theory of tensor multiplets assigned to adjoint representation of an internal symmetry group [38, 39].

${ }^{6}$ Explicitly, the number of components of an antisymmetric 3-tensor is $\frac{1}{6} N(N-1)(N-2)$ and of a symmetric traceless 3 -tensor is $\frac{1}{6} N(N-1)(N+4)$.
} 
triple M5-brane connections by "pants-like" membrane surfaces are providing the dominant contribution (pair-wise "cylinder" connections should give subleading $N^{2}$ contributions). ${ }^{7}$

Alternatively, one may think of an interacting $(2,0)$ tensor multiplet theory as a lowenergy limit of a tensionless $6 \mathrm{~d}$ string theory with closed strings carrying internal 3-plet indices which originate from virtual membranes connecting three parallel M5-branes: when the M5-branes get close, the membranes with 3 holes may effectively reduce to strings that then have 3 internal labels and thus $B_{\mu \nu}^{i j k}$ massless modes. The correlation between the 3 Lorentz indices and the 3 internal indices of the corresponding field strength $H_{\mu \nu \lambda}^{i j k}=d B_{\mu \nu}^{i j k}$ is thus a direct analog of the fact that the YM field strength which is a rank 2 Lorentz tensor carries also 2 internal indices (takes values in adjoint representation).

Ignoring the self-duality constraint on $H_{\mu \nu \lambda}$ one may start with a free theory

$$
L_{0}=H_{\mu \nu \lambda}^{i j k} H_{\mu \nu \lambda}^{i j k}, \quad \delta B_{\mu \nu}^{i j k}=\partial_{[\mu} \epsilon_{\nu]}^{i j k},
$$

where $\epsilon_{\mu}^{i j k}$ is a gauge parameter. A speculative idea of how to generalize this to an interacting theory is to assume that the role of the would-be structure constants here should be played by the generalized field strength $\widehat{H}_{\mu \nu \lambda}^{i j k}$ itself, i.e. that a non-linear generalization of the transformation rule for $B$ in (1.1) should have the following structure

$$
\widehat{\delta} B_{\mu \nu}^{i j k}=\partial_{[\mu} \epsilon_{\nu]}^{i j k}+\widehat{H}_{\mu \rho \lambda}^{i j^{\prime} k^{\prime}} B_{\nu \rho}^{i^{\prime} j k^{\prime}} \epsilon_{\lambda}^{i^{\prime} j^{\prime} k}+\ldots, \quad \widehat{H}_{\mu \nu \lambda}^{i j k}=\partial_{[\mu} B_{\nu \lambda]}^{i j k}+\widehat{H}_{\mu \rho \kappa}^{i j^{\prime} k^{\prime}} B_{\nu \rho}^{i^{\prime} j k^{\prime}} B_{\lambda \kappa}^{i^{\prime} j^{\prime} k}+\ldots,
$$

where dots stand for terms with other possible contractions of indices. The full non-linear field strength $\widehat{H}$ should then be non-polynomial in $B$. Such couplings required to contract 3-plet internal indices may have a generalization to self-dual case and may correspond to a "soft" gauge algebra structure (thus possibly avoiding no-go arguments against the existence of an interacting chiral antisymmetric tensor theory in [43, 44]).

One consequence of this 3-index assumption is that the leading interaction between the $B_{\mu \nu}^{i j k}$-fields should be quartic [30] rather than cubic as in the adjoint-representation YM theory

$$
L=\widehat{H}_{\mu \nu \lambda}^{i j k} \widehat{H}_{\mu \nu \lambda}^{i j k} \rightarrow \partial B^{i j k} \partial B^{i j k}+B^{i j k} B^{i j^{\prime} k^{\prime}} \partial B^{i^{\prime} j k} \partial B^{i^{\prime} j^{\prime} k}+\ldots=\mathrm{G}(B) \partial B \partial B+\ldots
$$

Adding scalar fields of tensor multiplets one may expect to get similar non-linear interactions, e.g., through covariant derivative $D_{\mu} \phi^{i j k}=\partial_{\mu} \phi^{i j k}+\widehat{H}_{\mu \rho \kappa}^{i j^{\prime} k^{\prime}} B_{\rho \kappa}^{i^{\prime} j k^{\prime}} \phi^{i^{\prime} j^{\prime} k}+\ldots$ Super-

\footnotetext{
${ }^{7}$ The relevance of triple M5-brane connections by membranes with 3 boundaries was suggested in [21] in order to explain the $\sqrt{N_{1} N_{2} N_{3}}$ scaling of the entropy of the extremal 4-d black hole described by the 2555 intersecting M-brane configurations. Similar virtual triple connections are not dominant in the case of open strings ending on D-branes as 3-string interactions are subleading in string coupling. 3-hole "pants-like" configurations may be viewed as basic building blocks of $2 \mathrm{~d}$ membrane surfaces: any membrane surface ending on several M5-branes may be cut into "pants" and thus surfaces with more than 3 holes should give subleading contributions in membrane interaction strength. An indication that 3-plet M2-brane interactions may be relevant is that the $C_{3}$ form of $11 \mathrm{~d}$ supergravity naturally couples to M2-brane world volume while the $11 \mathrm{~d}$ supergravity action contains the cubic interaction term $C_{3} \wedge d C_{3} \wedge d C_{3}$. In the limit when M5-branes coincide and thus the M2-brane configuration connecting them becomes small with string-like boundary, the membrane coupling $\int C_{3}$ should lead to $\int B_{2}$ coupling at the boundary of a membrane and this 2 -form should then carry $(i j k)$ indices. This is analogous to how the $B_{2}$-field coupling to world volume of a string translates into vector $\int A^{i j}$ couplings at the two boundary points of an open string connecting two D-branes.
} 
symmetry may require also quartic and higher self-interactions of the 3-plet scalars and fermions.

Even assuming such a hypothetical interacting $(2,0)$ tensor multiplet theory may exist at the classical level, one faces several difficult questions. The canonical dimension of the free $B$-field in $6 d$ is 2 and thus $H$ has dimension 3. Then the classical interactions in (1.2), (1.3) require a dimensional coupling parameter and thus break the classical conformal invariance of the free theory (1.1). One is then to hope that at the quantum level there may exist a non-trivial conformal fixed point at which the dimension of $B$ becomes 0 . Another important question is about an existence of a well-defined large $N$ limit in such theory. ${ }^{8}$ The multiple M5-brane theory should certainly admit a large $N$ expansion, as suggested, e.g., by the presence of 11d M-theory corrections to its anomalies [24, 29, 31, 32] and its free energy [25] which are subleading in $N$ compared to the leading $N^{3}$ term.

As a starting point, one may consider just a free 3-plet tensor multiplet CFT which correctly describes the $N^{3}$ term in the anomalies and protected 3-point functions as predicted by the $11 \mathrm{~d}$ supergravity. Regardless of its precise connection to multiple M5-brane theory, it should have a consistent $\mathrm{AdS}_{7}$ dual on its own right. Our aim below will be to study the thermal partition function in such free tensor multiplet theory with fields in a 3 -index representation of an internal symmetry group.

\subsection{Structure of the paper}

Below we shall mostly concentrate on the $S^{1} \times S^{d-1}$ partition function a free scalar field in a 3-fundamental representation of $\mathrm{U}(N)$. The cases of symmetric or antisymmetric 3-plet representation, $O(N)$ symmetry and 3-tensors with distinguishable indices will be similar. The generalization to free fermions or $\left(\frac{d}{2}-1\right)$-form fields and thus, in particular, to a $(2,0)$ tensor multiplet will be straightforward.

As we shall review in section 2, this partition function encodes the spectrum of "singletrace" primary operators in the free CFT. The singlet constraint may be implemented as in the familiar vector or adjoint representation cases by coupling the 3-plet field to a flat $\mathrm{U}(N)$ connection and integrating over its non-trivial holonomy on $S^{1}$, i.e. over a constant matrix $U \in \mathrm{U}(N)$. For a free field $\Phi$ in a general real representation $R$ of $\mathrm{U}(N)$ the resulting expression for the partition function $Z(x), x=e^{-\beta}$ will be given by the matrix $U$ integral in (2.7) with the "action" in the exponential depending on the character $\chi_{R}(U)$ and the one-particle partition function $z_{\Phi}(x)$.

In section 3 we shall compute the low temperature (small $x$ ) expansion of $Z$ in the large $N$ limit. We shall first expand the integral (2.7) in powers of $x$ at finite $N$ and then take $N \rightarrow \infty$. While in the familiar cases of the vector and adjoint representations the low temperature, $N=\infty$ expansion are convergent (and thus the $x \rightarrow 0$ and $N \rightarrow \infty$ limits commute in the low temperature phase $T \leq T_{c}$ ), in the 3 -plet case this expansion

\footnotetext{
${ }^{8}$ In 3-tensor models with distinguishable indices (i.e. invariant under the $[O(N)]^{3}$ symmetry) the large $N$ limit may be described by iterated "melonic" graphs [33-35, 37]. As was noted in [33], in the case when all 3 internal indices of an interacting $\phi^{4}$ scalar theory are indistinguishable, i.e. transform, e.g., in symmetric representation of a single $O(N)$ group, a "melonic" large $N$ limit may still exist but the convergence of the large $N$ expansion is unclear. We thank I. Klebanov and G. Tarnopolsky for clarifying comments on this issue.
} 
will be only asymptotic. The reason for this will be a rapid growth of the number of singlet operators with their dimension which will lead to the vanishing of the radius of convergence $x_{c}$ or the critical temperature $T_{c}$ in the $N=\infty$ limit. We shall find a closed expression (3.32) for the $N=\infty$ limit of the small $x$ expansion of $Z$ that encodes the number of singlet operators built out of elementary 3-plet fields to any order in dimension.

The analysis of the phase structure of $Z(x)$ at large but finite $N$ will be carried out in 4 . We shall rewrite the integral representation for $Z$ in terms of the eigenvalue density and study the large $N$ stationary-point solution for it. We shall find that in the 3-plet case there is a phase transition at the critical temperature $T_{c} \sim \frac{1}{\log N}$ which approaches zero at $N \rightarrow \infty$. At finite $N$ there are always two phases, $0<T \leq T_{c}$ and $T>T_{c}$, while at $N=\infty$ the first phase becomes essentially the $T=0$ one, so there is only the second phase for any $T>0 .{ }^{9}$ As in the vector and adjoint cases, we will find that in the $T>T_{c}$ phase the large $N$ stationary point solution for the eigenvalue density leads to $\log Z=\mathcal{O}\left(N^{2}\right)$.

Some concluding remarks about open issues will be made in section 5 . Few technical details and extensions will be presented in appendices. In particular, the case of 2-plet representation will be discussed in appendix A. The $O(N)$ case will be considered in appendix D. In appendix C.2 we shall compute the value of the eigenvalue density action on the stationary-point solution in the 3-plet case. In appendix E we shall give a general relation for the "single-trace" partition function counting only irreducible contractions in terms of the full $Z$.

In appendix C.4 we shall analyze the singlet partition function of a $p$-tensor with all indices being different, i.e. transforming under a separate copy of $\mathrm{U}(N)$ as in the tensor models in $[33,37]$. It turns out that the case of such $[\mathrm{U}(N)]^{p}$ theory is very similar to the $\mathrm{U}(N)$ one discussed above, with $p=3$ being again the critical value when the small temperature expansion becomes only asymptotic in the large $N$ limit, i.e. with the critical temperature being again $T_{c}(N \rightarrow \infty) \rightarrow 0$.

\section{Partition function with singlet constraint}

Given a CFT, one may be interested (in particular, in the context of AdS/CFT duality) in its thermal partition function $Z$ with a singlet constraint (see, e.g., [10, 13, 15-17, 45]). We shall consider a free field $\Phi$ in $S_{\beta}^{1} \times S^{d-1}$ transforming in a representation of the global symmetry group. The singlet projection may be implemented by coupling $\Phi$ to a flat connection $A_{\mu}=U^{-1} \partial_{\mu} U$ and integrating over it. Only the constant part of the $A_{0}$ component cannot be gauged away because of the non-trivial holonomy along the thermal circle. The resulting partition function $Z$ is then given by the 1-loop determinant with $A_{0^{-}}$ dependent covariant derivatives integrated over the constant eigenvalues of $A_{0}[10,15]{ }^{10}$ This gives an equivalent result to the coherent-state approach of $[13,16]$.

\footnotetext{
${ }^{9}$ This is similar to what happens in the $1 \mathrm{~d}$ Ising model where for $T=0$ there is no entropy term in the free energy and ordered phase is favored.

${ }^{10} \mathrm{See}$ also section 3 in [46] for a discussion of the case when $\Phi$ is a $4 \mathrm{~d}$ Maxwell field and $R$ is a vector representation of $\mathrm{U}(N)$ or $O(N)$ group.
} 
In general, the partition function on $S_{\beta}^{1} \times S^{d-1}$ may be written as

$$
Z=\sum_{\text {singlets }} x^{E}, \quad x=e^{-\beta},
$$

where we assume that the spatial sphere $S^{d-1}$ has a unit radius and $\beta=1 / T$. In a CFT, the discrete energy levels $E$ of states on $S^{d-1}$ can be identified with dimensions $\Delta$ of the corresponding operators in $\mathbb{R}^{d}$. Before singlet projection, physical states are obtained by acting on the vacuum with suitable composite operators built out of the elementary field $\Phi$. One may define the single-particle partition function

$$
z_{\Phi}(x)=\sum_{i} x^{E_{i}}
$$

that counts all such states treating $\Phi$ as a singlet, i.e. enumerates the components of $\Phi$ and its derivative descendants modulo free equations of motion (thus having also the interpretation of the character of the corresponding representation of the conformal group). For example, in the case of a free CFT represented by a scalar or Weyl (or Majorana) fermion in dimension $d$ and a vector in $4 \mathrm{~d}$ or a self-dual rank 2 tensor in $6 \mathrm{~d}$ one finds the well known expressions (see, e.g., [47, 48])

$$
\begin{aligned}
z_{\mathrm{S}, d}(x) & =\frac{x^{\frac{d}{2}-1}(1+x)}{(1-x)^{d-1}}, & z_{\mathrm{F}, d}(x) & =\frac{2^{\frac{d}{2}} x^{\frac{d-1}{2}}}{(1-x)^{d-1}}, \\
z_{\mathrm{V}, 4}(x) & =\frac{6 x^{2}-2 x^{3}}{(1-x)^{3}}, & z_{\mathrm{T}, 6}(x) & =\frac{10 x^{3}-5 x^{4}+x^{5}}{(1-x)^{5}} .
\end{aligned}
$$

For a multiplet of free conformal fields one is to combine properly the contributions to $Z$ coming from $z_{\Phi}$ for individual fields (cf. (2.7), (2.8)). ${ }^{11}$

Let us focus on the simplest case of a single bosonic field $\Phi$ transforming in a real representation $R$ of $\mathrm{U}(N) .{ }^{12}$ The full partition function $Z$ may be expressed as a sum over

\footnotetext{
${ }^{11}$ For example, if one formally sums up the single-particle partition functions in (2.3), (2.4) taking the fermion contribution with the plus sign one finds for the $4 \mathrm{~d} \mathcal{N}=4$ Maxwell multiplet and $6 \mathrm{~d}(2,0)$ tensor multiplet [31]

$$
\begin{aligned}
& z_{\mathcal{N}=4}=6 z_{\mathrm{S}, 4}+4 z_{\mathrm{F}, 4}+z_{\mathrm{V}, 4}=\frac{6 x+16 x^{\frac{3}{2}}+12 x^{2}-2 x^{3}}{(1-x)^{3}}, \\
& z_{(2,0)}=5 z_{\mathrm{S}, 6}+2 z_{\mathrm{F}, 6}+z_{\mathrm{T}, 6}=\frac{5 x^{2}+16 x^{\frac{5}{2}}+15 x^{3}-5 x^{4}+x^{5}}{(1-x)^{5}} .
\end{aligned}
$$

Note that these combinations actually appear in the full partition function $(2.7)$ only in one $(m=1)$ of the terms as the fermionic contribution enters with the sign $(-1)^{m+1}$ depending on the term in the infinite sum in the exponent.

${ }^{12}$ If $\Phi$ is complex, $R$ will be the representation acting on the real components. For example, if $\Phi_{i}$ transforms in the fundamental representation of $\mathrm{U}(N), \Phi_{i}^{\prime}=U_{i j} \Phi_{j}$, then $R=N \oplus \bar{N}$, i.e. if $\Phi_{i}=\alpha_{i}+i \beta_{i}$ and $U=A+i B$, then $\left(\begin{array}{c}\alpha_{i}^{\prime} \\ \beta_{i}^{\prime}\end{array}\right)=\mathscr{U}\left(\begin{array}{c}\alpha_{i} \\ \beta_{i}\end{array}\right)=\left(\begin{array}{cc}A & -B \\ B & A\end{array}\right)\left(\begin{array}{c}\alpha_{i} \\ \beta_{i}\end{array}\right)$. This is a reducible $2 N$ dimensional representation that may be identified with $N \oplus \bar{N}$. Its character is

$$
\operatorname{tr} \mathscr{U}=2 \operatorname{tr} A=\operatorname{tr}\left(U+U^{*}\right)=\operatorname{tr}(U)+\operatorname{tr}\left(U^{-1}\right)=\chi_{N}(U)+\chi_{\bar{N}}(U) .
$$
}


the occupation numbers of all modes, with a Boltzmann factor $e^{-\beta E}$ for the total energy $E$ and a numerical factor counting the number of singlets in the corresponding product of representations. This gives

$$
Z=\sum_{n_{1} \geq 0} x^{n_{1} E_{1}} \sum_{n_{2} \geq 0} x^{n_{2} E_{2}} \ldots\left\{\# \text { of singlets in } \operatorname{sym}^{n_{1}}(R) \otimes \operatorname{sym}^{n_{2}}(R) \ldots\right\}
$$

The number of singlets is obtained by integrating over the global symmetry group with the invariant Haar measure $d U$ (normalized as $\int d U=1$ )

$$
Z=\int d U \prod_{i} \sum_{n_{i} \geq 0} x^{n_{i} E_{i}} \chi_{\mathrm{sym}^{n_{i}}\left(R_{i}\right)}(U)
$$

Using the explicit form of the character $\chi$ of the symmetric power $\operatorname{sym}^{n}(R)$ (see, e.g., eq. (A.8) in [15]), we can then write the singlet partition function as $[13,16]$

$$
Z=\int d U \exp \left\{\sum_{i} \sum_{m=1}^{\infty} \frac{1}{m} x^{m E_{i}} \chi_{R}\left(U^{m}\right)\right\}=\int d U \exp \left\{\sum_{m=1}^{\infty} \frac{1}{m} z_{\Phi}\left(x^{m}\right) \chi_{R}\left(U^{m}\right)\right\}
$$

Here we assumed that $\Phi$ is a boson; in the mixed boson $(\mathrm{B})+$ fermion $(\mathrm{F})$ case we need to do the replacement

$$
z_{\Phi}\left(x^{m}\right) \rightarrow z_{B}\left(x^{m}\right)+(-1)^{m+1} z_{F}\left(x^{m}\right) .
$$

Below we shall consider mainly the following representations $R$ (with characters $\chi_{R}$ )

\begin{tabular}{|cc|}
\hline$R$ & $\chi_{R}$ \\
\hline vector : $N \oplus \bar{N}$ & $\operatorname{tr}(U)+\operatorname{tr}\left(U^{-1}\right)$ \\
adjoint : & $\operatorname{tr}(U) \operatorname{tr}\left(U^{-1}\right)$ \\
3-plet : $N^{\otimes 3} \oplus \bar{N}^{\otimes 3}$ & {$[\operatorname{tr}(U)]^{3}+\left[\operatorname{tr}\left(U^{-1}\right)\right]^{3}$} \\
\hline
\end{tabular}

In general, for the $p$-plet field transforming in the product of $p$ fundamental representations when $R=N^{\otimes p} \oplus \bar{N}^{\otimes p}$ one finds

$$
\chi_{N \otimes p} \bar{N}^{\otimes p}(U)=[\operatorname{tr}(U)]^{p}+\left[\operatorname{tr}\left(U^{-1}\right)\right]^{p} .
$$

One may also be interested in the antisymmetric or symmetric tensor representations. For example, in the case of the 3 -plet representation one finds

$$
\chi_{\left(R_{0} \otimes R_{0} \otimes R_{0}\right)_{(\text {anti) sym }}}(U)=\frac{1}{6}\left[\chi_{R_{0}}(U)\right]^{3} \pm \frac{1}{2} \chi_{R_{0}}(U) \chi_{R_{0}}\left(U^{2}\right)+\frac{1}{3} \chi_{R_{0}}\left(U^{3}\right),
$$

where $R_{0}$ is a fundamental or anti-fundamental representation, and sign (-)+ applies to (anti) symmetric case. 


\section{$3 \quad N=\infty$ limit of low temperature expansion of $U(N)$ partition function}

In the $N=\infty$ limit the counting of states is expected to simplify because singlets can be constructed without considering special features of the finite $N$ case $[13,14]$. In general, $Z$ may be expressed in terms of the "single-trace" partition function $Z_{\text {s.t. }}(x)$ - a generating function of fully connected (indecomposable) contractions of fields

$$
\log Z(x) \equiv \sum_{m=1}^{\infty} \frac{1}{m} Z_{\text {s.t. }}\left(x^{m}\right) .
$$

The expression for $Z_{\text {s.t. }}(x)$ is well known in the vector and adjoint representation cases and can be generalized to higher representations. As we shall discuss in appendix E, one can formally invert the relation (3.1) and determine $Z_{\text {s.t. }}(x)$ in terms of $Z(x)$.

Below, in section 3.1, we shall review the known expressions for the $N=\infty$ partition functions in the vector and adjoint cases and then in section 3.2 turn to the 3-plet case. We shall first consider the expansion of the 3-plet $Z$ in (2.7) in small $x$ for finite $N$ and then take the $N \rightarrow \infty$ limit of the coefficients at each order in $x$. In contrast to the vector and adjoint cases, here the small $x$ and large $N$ limits will not commute: the large $N$ limit of the $x^{n}$ coefficients in $Z$ will grow too fast with $n$ so that the small $x$ expansion will not converge, i.e. the radius of convergence goes to zero in the $N \rightarrow \infty$ limit.

The reason for that will be understood in section 4 where will find that there is a phase transition at the critical temperature $T_{c} \sim(\log N)^{-1}$ which goes to zero when $N \rightarrow \infty$. Thus the low temperature phase effectively disappears (shrinks to $T=0$ ) in the strict $N=\infty$ limit.

In section 3.3 we shall present an exact expression that summarizes the asymptotic low temperature expansion of the $N \rightarrow \infty 3$-plet partition function. This closed form encodes the number of singlet operators built out of elementary 3-plet fields at any order in dimension.

\subsection{Vector and adjoint cases}

In the vector representation case the singlets in $\operatorname{sym}^{n}(N \oplus \bar{N})$ in $(2.7)$ are products of invariant bilinears in operators built out of $\Phi$ and $\bar{\Phi}$. For example, the bilinear singlets built out of a scalar field have the form $\sum_{s s^{\prime}} c_{s s^{\prime}} \sum_{i} \partial^{s} \bar{\Phi}_{i} \partial^{s^{\prime}} \Phi_{i} .{ }^{13}$ The partition function of such bilinears is the square of the single-particle partition function (2.2), i.e. the "singletrace" partition function here is

$$
Z_{\mathrm{s.t.}}^{\mathrm{vector}}(x)=\left[z_{\Phi}(x)\right]^{2} .
$$

Including products of the bilinears, i.e. of all possible singlets, we get the $N=\infty$ partition function for the vector representation in the form (3.1) [10]

$$
\log Z^{\mathrm{vector}}=\sum_{m=1}^{\infty} \frac{1}{m}\left[z_{\Phi}\left(x^{m}\right)\right]^{2} .
$$

\footnotetext{
${ }^{13}$ There are also singlets built using the invariant $\epsilon_{i_{1} \ldots . i_{N}}$ tensor but their effect on the partition function is exponentially suppressed at large $N[10]$.
} 
In the adjoint case the singlets are built as products of single-trace operators. The partition function for single-trace operators may be found by the Polya enumeration theorem $[13,14]$

$$
Z_{\text {s.t. }}^{\text {adjoint }}=-\sum_{m=1}^{\infty} \frac{\varphi(m)}{m} \log \left[1-z_{\Phi}\left(x^{m}\right)\right] .
$$

Here $\varphi(m)$ is the Euler's totient function counting all positive integers up to a given integer $m$ that are relatively prime to $m$. The full partition function is obtained by considering multi-trace singlets treating single trace states as identical particles. As a result, the $N=\infty$ partition function in the adjoint case is given by

$$
\log Z^{\text {adjoint }}=\sum_{m=1}^{\infty} \frac{1}{m} Z_{\text {s.t. }}^{\text {adjoint }}\left(x^{m}\right)=-\sum_{m=1}^{\infty} \log \left[1-z_{\Phi}\left(x^{m}\right)\right],
$$

As was already mentioned above (see (2.8)), in the general boson plus fermion field case one has to replace $z_{\Phi}\left(x^{m}\right)$ by $z_{B}\left(x^{m}\right)+(-1)^{m+1} z_{F}\left(x^{m}\right)$ in (3.3) and (3.5).

From the AdS/CFT perspective, the bilinears in the vector case are in direct correspondence with the massless higher spin fields in AdS. Hence the total partition function (3.3) should match the AdS partition function [4, 10, 12]. Similarly, in the adjoint case, the single trace operators correspond to the spectrum massless and massive higher spin fields in AdS. On the group-theoretical basis, one expects again to match the full multi-particle partition function (3.5) with its AdS counterpart [7-9].

\subsection{3 -plet case: low temperature expansion of $Z$ and counting of operators}

For fields transforming in a generic representation direct counting of states may be very cumbersome. One may work out the direct expansion of the partition function (2.7) in powers of $x=e^{-\beta}$ that effectively encodes the counting of singlet operators. Taking into account the specific form of the characters (cf. (2.9)) one then needs to compute the $\mathrm{U}(N)$ group integrals

$$
I(\boldsymbol{a}, \boldsymbol{b})=\int d U \prod_{\ell \geq 1}\left(\operatorname{tr} U^{\ell}\right)^{a_{\ell}} \overline{\left(\operatorname{tr} U^{\ell}\right)^{b_{\ell}}}
$$

where $\boldsymbol{a}=\left(a_{\ell}\right)$ and $\boldsymbol{b}=\left(b_{\ell}\right)$ are sets of integers. Such integrals can be found straightforwardly for finite $N$, but the computational complexity grows rapidly with increasing $N$. There are efficient techniques to improve the computation, see for instance [49, 50]. Here we are interested in the large $N$ limit. The integrals (3.6) are zero if

$$
\kappa(\boldsymbol{a}) \equiv \sum_{\ell} \ell a_{\ell} \neq \sum_{\ell} \ell b_{\ell}
$$

and do not depend on $N$ as soon as $\kappa(\boldsymbol{a}) \leq N$, see [51]. At each order in small $x$ expansion we find a finite set of integrals $I(\boldsymbol{a}, \boldsymbol{b})$ and the above condition is certainly true if $N$ is sufficiently large, i.e. it is not actually necessary to consider the case of $N=\infty$ directly. One then finds the following simple result

$$
I(\boldsymbol{a}, \boldsymbol{b})=\prod_{\ell \geq 1} \ell^{a_{\ell}} a_{\ell} ! \delta_{a_{\ell}, b_{\ell}} .
$$


Let us consider, for example, the case when the field $\Phi$ is a $4 \mathrm{~d}$ scalar with the one-particle partition function $z_{\Phi}(x)=z_{\mathrm{S}, 4}(x)$ given in (2.3). If the scalar is in the vector representation of $\mathrm{U}(N)$, expanding $Z$ in (2.7) in powers of $x \ll 1$ one finds

$$
\begin{aligned}
Z_{\mathrm{S}, 4}^{\text {vector }}=1+\int d U & {\left[x^{2} \tau_{1}+8 x^{3} \tau_{1}+x^{4}\left(34 \tau_{1}+\frac{1}{4} \tau_{1}^{2}+\frac{1}{4} \tau_{2}\right)+x^{5}\left(104 \tau_{1}+4 \tau_{1}^{2}\right)\right.} \\
& \left.+x^{6}\left(259 \tau_{1}+33 \tau_{1}^{2}+\frac{1}{36} \tau_{1}^{3}+2 \tau_{2}+\frac{1}{4} \tau_{1} \tau_{2}+\frac{1}{9} \tau_{3}\right)+\ldots\right],
\end{aligned}
$$

where $\tau_{\ell}(U) \equiv\left(\operatorname{tr} U^{\ell}\right) \overline{\left(\operatorname{tr} U^{\ell}\right)}$. Using (3.8) we find at $N \rightarrow \infty$

$$
Z_{\mathrm{S}, 4}^{\mathrm{vector}}=1+x^{2}+8 x^{3}+35 x^{4}+112 x^{5}+330 x^{6}+944 x^{7}+2849 x^{8}+\ldots .
$$

The coefficient of a given power $x^{k}$ stabilizes as soon as $N>N_{k}$ with $N_{k}$ growing with $k$. Thus the expansion derived up to $x^{k}$ is exact as soon as $N>\max \left\{N_{k}\right\}$ which is a finite number. We observe that (3.10) agrees with the small $x$ expansion of (3.3) with $z_{\Phi}=z_{\mathrm{S}, 4}$ in (2.3). Note that (3.3) was derived directly at $N=\infty$. The reason for the agreement is that here the $N \rightarrow \infty$ and $x \rightarrow 0$ limits commute. This is reflected in convergence of the series in (3.10) which is also related to the fact that the critical temperature at which the low temperature phase is no longer valid here goes to infinity at large $N$ (see (4.3)).

Similarly, in the adjoint case we recover the expansion of (3.5), i.e.

$$
Z_{\mathrm{S}, 4}^{\text {adjoint }}=1+x+6 x^{2}+20 x^{3}+75 x^{4}+252 x^{5}+914 x^{6}+3160 x^{7}+11194 x^{8}+\ldots .
$$

The series (3.11) have a finite radius of convergence which reflects the fact that in the adjoint case the critical temperature above which the low temperature phase no longer exists is of order 1 (see (4.4)).

Let us also recall how the direct counting of operators goes in the vector and adjoint $\mathrm{U}(N)$ cases. For the vector representation, the "single-trace" (fully connected) partition function in (3.1), (3.3) is $\left[z_{\mathrm{S}, 4}(x)\right]^{2}=x^{2}+8 x^{3}+\ldots$ This corresponds to one operator at dimension $2 \bar{\varphi}_{i} \varphi_{i}$ and the $4+4$ operators $\bar{\varphi}_{i} \partial_{\mu} \varphi_{i}$ and $\partial_{\mu} \bar{\varphi}_{i} \varphi_{i}$ at dimension 4 . In the adjoint case, the single-trace partition function (3.4) for a $4 \mathrm{~d}$ scalar is $Z_{\mathrm{s.t} .}^{\text {adjoint }}=x+5 x^{2}+\ldots$ The coefficients in this expansion correspond to one operator $\operatorname{tr}(\varphi)$ of dimension 1 and $1+4=5$ operators $\operatorname{tr}\left(\varphi^{2}\right)$ and $\partial_{\mu} \operatorname{tr}(\varphi)$ of dimension 2 .

In the novel 3-plet representation case we find from $(2.7),(3.9)(3.8)$ that the $4 \mathrm{~d}$ scalar partition function has the following large $N$ limit of its small $x$ expansion (see also appendix B for some finite $N$ data)

$$
\begin{aligned}
Z_{\mathrm{S}, 4}^{3 \text {-plet }}= & 1+6 x^{2}+48 x^{3}+396 x^{4}+3504 x^{5}+35580 x^{6}+381216 x^{7}+4408956 x^{8} \\
& +53647632 x^{9}+689785308 x^{10}+9258337104 x^{11}+129842959752 x^{12} \\
& +1889221738416 x^{13}+\ldots
\end{aligned}
$$

It is easy to see how the first three terms here may be reproduced by counting the singlet operators. At dimension 2, we have the singlets built out of a scalar $\Phi=\left(\varphi_{i j k}\right)$ of the structure

$$
(\bar{\varphi} \varphi)
$$


i.e. fully connected contractions $\bar{\varphi}_{i j k} \varphi_{i^{\prime} j^{\prime} k^{\prime}}$ where $i^{\prime} j^{\prime} k^{\prime}$ is a permutation of $i j k$. This gives $3 !=6$ different cases. At dimension 3 , we have the singlets

$$
\left(\bar{\varphi} \partial_{\mu} \varphi\right), \quad\left(\partial_{\mu} \bar{\varphi} \varphi\right)
$$

with the same contractions as above. This gives total of $2 \times 4 \times 6=48$ of dimension 3 operators. At dimension 4 we get the bilinear structures

$$
\left(\bar{\varphi} \partial_{\mu} \partial_{\nu} \varphi\right), \quad\left(\partial_{\mu} \partial_{\nu} \bar{\varphi} \varphi\right), \quad\left(\partial_{\mu} \bar{\varphi} \partial_{\nu} \varphi\right)
$$

Ignoring terms vanishing on the equation of motion $\partial^{\mu} \partial_{\mu} \varphi=0$ gives $(9 \times 2+4 \times 4) \times 6=204$ operators. Then there is the quartic structure

$$
(\bar{\varphi} \varphi)(\bar{\varphi} \varphi),
$$

where we may have 6 contraction in each factor; counting only once the symmetric cases, we get $\frac{1}{2} \times 6 \times 7=21$ operators. Finally, there is also an irreducible "single-trace" operator

$$
(\bar{\varphi} \varphi \bar{\varphi} \varphi) .
$$

Here each $\bar{\varphi} \varphi$ appears in the form $\bar{\varphi}_{i j k} \varphi_{i j l}=X_{k l}$ where the free indices may be in any position and the two pairs of contractions may be in each of the two possibilities. This gives $3^{2} \times 2=18$ ways of constructing $X$. Contracting the two $X$ 's (counting once the symmetric cases) gives $\frac{1}{2} \times 18 \times 19=171$ operators. The total number of dimension 4 singlet operators is then $204+21+171=396$, in agreement with the coefficient of the $x^{4}$ term in (3.12). ${ }^{14}$

We may also find the corresponding "single-trace" generating function of fully connected contractions by writing (3.12) in the form (3.1)

$$
\begin{aligned}
Z_{\mathrm{s.t.}}^{3-\text { plet }}= & 6 x^{2}+48 x^{3}+375 x^{4}+3216 x^{5}+32098 x^{6}+342912 x^{7}+3976443 x^{8} \\
& +48645632 x^{9}+629746974 x^{10}+8512245744 x^{11} \\
& +120220813597 x^{12}+1760740453968 x^{13}+\ldots
\end{aligned}
$$

The partition function (3.12) corresponds to a $4 \mathrm{~d}$ scalar in the general 3-index tensor representation of $\mathrm{U}(N)$ without any symmetry. In the case of totally symmetric (+) or antisymmetric (-) representations we find, using the expressions for the characters in (2.11)

$$
\begin{aligned}
Z_{\mathrm{S}, 4}^{3 \text {-plet }^{+}=} & 1+x^{2}+8 x^{3}+36 x^{4}+120 x^{5}+404 x^{6}+1368 x^{7}+5034 x^{8} \\
& +18736 x^{9}+71452 x^{10}+276864 x^{11}+\ldots, \\
Z_{\mathrm{S}, 4}^{3-\text { plet }^{-}=} & 1+x^{2}+8 x^{3}+36 x^{4}+120 x^{5}+403 x^{6}+1360 x^{7}+4978 x^{8} \\
& +18400 x^{9}+69645 x^{10}+267728 x^{11}+\ldots
\end{aligned}
$$

\footnotetext{
${ }^{14}$ Let us note that if we formally replace $z_{\Phi}(x)$ by $x$ in $(2.7)$, that will correspond to considering a constant complex $4 \mathrm{~d}$ scalar field that has dimension 1 (cf. $z_{\mathrm{S}, 4}$ in $(2.3)$ ). In this case $Z$ will count just the $\mathrm{U}(N)$ contractions leading to singlets. This counting has been worked out in [52]. In the 3-plet representation case one obtains the series $Z=1+6 x^{2}+192 x^{4}+10170 x^{6}+834612 x^{8}+90939630 x^{10}+12360636540 x^{12}+\ldots$, which agrees with eq. (86) of [52]. The $192 x^{4}$ term comes from the sum of the 21 structures in (3.16) and the 171 in (3.17) that do not involve derivatives.
} 
Compared to (3.12) we see that there there are fewer operators as some of the contractions are now equivalent. Note that the partition functions (3.19) and (3.20) start to differ at order $\mathcal{O}\left(x^{6}\right)$. The coefficients of the first few terms in (3.19) or (3.20) can be easily reproduced by the operator counting. ${ }^{15}$

Similarly, in the case of a scalar in the 3-plet representations in 6 dimensions we find the following analogs of (3.12), (3.19), (3.20)

$$
\begin{aligned}
Z_{\mathrm{S}, 6}^{3-\text { plet }}= & 1+6 x^{4}+72 x^{5}+456 x^{6}+2040 x^{7}+7452 x^{8}+26232 x^{9} \\
& +111768 x^{10}+591432 x^{11}+3268332 x^{12}+16860144 x^{13}+\ldots, \\
Z_{\mathrm{S}, 6}^{3-\text { plet }^{+}=} & 1+x^{4}+12 x^{5}+76 x^{6}+340 x^{7}+1212 x^{8}+3676 x^{9} \\
& +10032 x^{10}+25956 x^{11}+68632 x^{12}+196788 x^{13}+\ldots, \\
Z_{\mathrm{S}, 6}^{3-\text { plet }^{-}}= & 1+x^{4}+12 x^{5}+76 x^{6}+340 x^{7}+1212 x^{8}+3676 x^{9} \\
& +10032 x^{10}+25956 x^{11}+68631 x^{12}+196776 x^{13}+\ldots
\end{aligned}
$$

As a $6 \mathrm{~d}$ scalar has dimension 2 , the low temperature expansion here starts at $x^{4}$ order. The (anti) symmetric 3-plet partition functions (3.22), (3.23) differ at $\mathcal{O}\left(x^{12}\right)$, i.e. at the level of operators with three $\bar{\varphi} \varphi$ pairs; this is in parallel with what was in $4 \mathrm{~d}$ where the scalar dimension was 1 (eqs. (3.19), (3.20) differ at $x^{6}$ order). Comparing the coefficients in the vector (3.10), adjoint (3.11) and 3-plet cases (3.12) we conclude that the number of singlet operators in the 3 -plet case grows much faster with the power of $x$, i.e. with the operator dimension. This implies non-convergence of the small $x$ expansion. Indeed, as we shall find in section 4 an analog of the "Hagedorn" transition found in the adjoint case [5, 15] here happens at much lower temperature $T_{c} \sim(\log N)^{-1}$ which goes to zero at $N \rightarrow \infty$. This will also become clear from the closed expression for the $N=\infty$ limit of the small $x$ partition function presented in the subsection 3.3.

One may also find similar low temperature expansions of $Z$ in the case when the global symmetry group is $O(N)$ instead of $\mathrm{U}(N)$; this is discussed in appendix D.

\subsection{Exact expression for low temperature expansion of 3-plet partition func- tion in the $N \rightarrow \infty$ limit}

Given a particular choice of the character $\chi_{R}$ in (2.7) it is possible to find a closed form for the small $x$ expansion of the partition function extending the expansions like (3.12) to all orders in $x$.

\footnotetext{
${ }^{15}$ The terms $x^{2}+8 x^{3}$ are the same as as in the vector case (3.10) as the correspond to the operators (3.13) and (3.14) without additional multiplicity as the 3-indices are now contracted in a unique way $\left(\bar{\varphi}_{i j k} \varphi_{i j k}\right.$, etc.). The coefficient 36 of the $x^{4}$ term corresponds to 34 dimension 4 operators in (3.15) (now with unique contraction), one operator as in (3.16) of the form $\left(\bar{\varphi}_{i j k} \varphi_{i j k}\right)^{2}$, and one additional operator $\bar{\varphi}_{i j k} \varphi_{i j l} \bar{\varphi}_{p q l} \varphi_{p q k}$ as in (3.17). For completeness, let us list also the "single-trace" partition functions corresponding to (3.19), (3.20):

$$
\begin{aligned}
& Z_{\text {s.t. }}^{3-\text { plet }^{+}}=x^{2}+8 x^{3}+35 x^{4}+112 x^{5}+332 x^{6}+968 x^{7}+3104 x^{8}+10672 x^{9}+39466 x^{10}+153160 x^{11}+\ldots \\
& Z_{\text {s.t. }}^{3-\text { plet }^{-}}=x^{2}+8 x^{3}+35 x^{4}+112 x^{5}+331 x^{6}+960 x^{7}+3049 x^{8}+10352 x^{9}+37814 x^{10}+145192 x^{11}+\ldots
\end{aligned}
$$
}


Let us start from the following general form of the series expansion of $(2.7)^{16}$

$$
Z=\prod_{m=1}^{\infty} \sum_{k=0}^{\infty} \frac{1}{k !}\left(\frac{z_{\Phi}\left(x^{m}\right)}{m}\right)^{k} \int d U\left[\chi_{R}\left(U^{m}\right)\right]^{k} .
$$

If we consider the $p$-plet (or $N^{\otimes p}$ representation of $\mathrm{U}(N)$, then the corresponding character is given by $(2.10)$, i.e. $\chi_{R}(U)=[\operatorname{tr}(U)]^{p}+\left[\operatorname{tr}\left(U^{-1}\right)\right]^{p}$, and the only non-vanishing contribution to (3.24) comes from the term with an equal number of $\operatorname{tr}(U)$ and $\operatorname{tr}\left(U^{-1}\right)$ factors. Hence, using (3.8), we find

$$
\begin{aligned}
Z^{p \text {-plet }} & =\prod_{m=1}^{\infty} \sum_{\substack{k=0 \\
k \text { even }}}^{\infty} \frac{1}{k !}\left(\frac{z_{\Phi}\left(x^{m}\right)}{m}\right)^{k}\left(\begin{array}{c}
k \\
k / 2
\end{array}\right) m^{p k / 2}(p k / 2) ! \\
& =\prod_{m=1}^{\infty} \sum_{k=0}^{\infty} \frac{1}{(2 k) !}\left(\frac{z_{\Phi}\left(x^{m}\right)}{m}\right)^{2 k}\left(\begin{array}{c}
2 k \\
k
\end{array}\right) m^{p k}(p k) ! \\
& =\prod_{m=1}^{\infty} F_{p}\left(m^{p-2}\left[z_{\Phi}\left(x^{m}\right)\right]^{2}\right),
\end{aligned}
$$

where we have introduced the formal power series

$$
F_{p}(y) \equiv \sum_{k=0}^{\infty} b_{k} y^{k}, \quad b_{k}=\frac{(p k) !}{(k !)^{2}}, \quad p=1,2,3, \ldots
$$

As a check, for $p=1$ and $p=2$ we find from (3.26):

$$
F_{1}(y)=e^{y}, \quad F_{2}(y)=\frac{1}{\sqrt{1-4 y}},
$$

and thus (3.25) gives

$$
\begin{aligned}
\log Z^{1 \text {-plet }} & =\sum_{m=1}^{\infty} \frac{1}{m}\left[z_{\Phi}\left(x^{m}\right)\right]^{2}, \\
\log Z^{2-\text { plet }} & =-\frac{1}{2} \sum_{m} \log \left(1-4\left[z_{\Phi}\left(x^{m}\right)\right]^{2}\right),
\end{aligned}
$$

which are indeed the expressions for the partition functions of the vector (3.3) and 2plet (A.3) representations (cf. also (E.8)). Let us mention for completeness that in the adjoint case one finds, similarly to (3.25),

$$
Z^{\text {adjoint }}=\prod_{m=1}^{\infty} \sum_{k=0}^{\infty} \frac{1}{k !}\left(\frac{z_{\Phi}\left(x^{m}\right)}{m}\right)^{k} m^{k} k !=\prod_{m=1}^{\infty} \sum_{k=0}^{\infty}\left[z_{\Phi}\left(x^{m}\right)\right]^{k}=\prod_{m=1}^{\infty}\left[1-z_{\Phi}\left(x^{m}\right)\right]^{-1}
$$

which is indeed the partition function in (3.5).

\footnotetext{
${ }^{16}$ We exploit the fact that the integral over $U$ vanishes when evaluated on products of characters of $U^{m}$ with different values of $m$.
} 
Surprisingly, the series $F_{p}$ in (3.26) no longer converges (has zero radius of convergence $)^{17}$ starting with $p=3$. Thus for $p \geq 3$ (3.26) should be regarded only as a formal generating function. One may "resum" (3.26) by first replacing the numerator $(3 k)$ ! in $b_{k}$ in (3.26) by $\int_{0}^{\infty} d t e^{-t} t^{3 k}$, then summing over $k$ and finally integrating over $t$. In this way we obtain

$$
F_{3}(y) \rightarrow \widetilde{F}_{3}(y)=\frac{1}{6} \sqrt{\frac{\pi}{3} y^{-1}} e^{-\frac{1}{54} y^{-1}}\left[I_{\frac{1}{6}}\left(\frac{1}{54} y^{-1}\right)+I_{-\frac{1}{6}}\left(\frac{1}{54} y^{-1}\right)\right],
$$

where $I_{a}(y)$ is the modified Bessel function of the first kind. The function $\widetilde{F}_{3}(y)$ has a branch cut on the negative real axis, but is real, positive and smooth for $y \geq 0$. The power series (3.26) defining $F_{3}(y)$ is precisely an asymptotic expansion of $\widetilde{F}_{3}(y)$ for $y \rightarrow 0^{+}$, i.e. for any integer $K$ and $y \rightarrow 0^{+}$we have $\widetilde{F}_{3}(y)-\sum_{k=0}^{K} \frac{(3 k) !}{(k !)^{2}} y^{k}=\mathcal{O}\left(y^{K+1}\right) .{ }^{18}$

It thus follows from (3.25), (3.26) that, in particular, for a $4 \mathrm{~d}$ scalar field in the 3 -plet representation

$$
Z_{\mathrm{S}, 4}^{3 \text {-plet }}=\prod_{m=1}^{\infty} \sum_{k=0}^{\infty} \frac{(3 k) !}{(k !)^{2}} m^{k}\left[z_{\Phi}\left(x^{m}\right)\right]^{2 k}, \quad z_{\Phi}(x)=z_{\mathrm{S}, 4}(x)=\frac{x(1+x)}{(1-x)^{3}},
$$

which gives a closed expression for the all-order generalization of the leading terms in the expansion of $Z_{\mathrm{S}, 4}^{3-\text { plet }}$ in (3.12).

As follows from (3.25), the same general expression (3.32) applies also to other fields with the corresponding one-particle partition functions $z_{\Phi}$ (with statistics accounted for by (2.8)). It thus encodes the number of singlet operators built out of the field $\Phi$ in 3-plet representation of any integer dimension. Given $Z^{3 \text {-plet }}(x)$ and using the inversion formula in appendix E one can find also the corresponding single-trace partition function in (3.1) that counts the number of singlet operators represented by irreducible contractions.

Similar computation can be carried out in the case of a $p$-tensor field with $p$ distinguished indices transforming under separate $\mathrm{U}(N)$ groups: the singlet partition function $Z$ is then defined by gauging the full $[\mathrm{U}(N)]^{p}$ group (see [33]). In this case there are less singlet operators but again the large $N$ limit of the small $x$ expansion of $Z$ becomes only asymptotic starting with $p=3$ case (see appendix $\mathrm{F}$ ).

${ }^{17}$ This is implied by the growth of $\frac{b_{k+1}}{b_{k}}=\left.\frac{(k p+p) !}{(k+1)^{2}(k p) !}\right|_{p=3}=27 k+\frac{6}{1+k}$, with $b_{k}$ defined in (3.26).

${ }^{18}$ Alternatively, the Borel transform of $F_{3}(y)$ in $(3.26)$ is given by

$$
F_{3}^{B}(y) \equiv \sum_{k=0}^{\infty} \frac{b_{k}}{k !} y^{k}={ }_{2} F_{1}\left(\frac{1}{3}, \frac{2}{3}, 1,27 y\right)
$$

and thus the resulting Borel sum of $F_{3}(y)$ is expressed in terms of the Bessel $K$-function

$$
\widetilde{F}_{3}^{B}(y)=\int_{0}^{\infty} d t e^{-t} F_{B}(y t)=\frac{1}{3} \sqrt{-\frac{1}{3 \pi} y^{-1}} e^{-\frac{1}{54} y^{-1}} K_{\frac{1}{6}}\left(-\frac{1}{54} y^{-1}\right) .
$$

$\widetilde{F}_{3}^{B}(y)$ has an imaginary part for $y>0$ which vanishes exponentially fast for $y \rightarrow 0^{+}$and does not affect the asymptotic expansion of $\widetilde{F}_{3}^{B}(y)$ which is the same as $F_{3}(y)$ in (3.26). 


\section{Large $N$ partition function and phase transitions}

\subsection{Overview}

In the previous section we directly computed the $N=\infty$ limit of the small $x$ (small temperature) expansion of the partition function and observed the rapid growth of the number of states with the increase of dimension of the $\mathrm{U}(N)$ representation. This suggests a non-trivial dependence of $Z$ on the temperature with possible phase transitions.

Let us recall what happens in the well-known case of the adjoint representation. If the typical number of states relevant for thermodynamics at certain $\beta=1 / T$ is much smaller than $N^{2}$, then (3.5) is a good approximation to the exact partition function. This cannot be true at any temperature. While the expression (3.5) is well defined at low temperatures it diverges as soon as $z_{\Phi}(x)$ becomes of order 1 . On general grounds, we can prove that the equation $z_{\Phi}(x)=1$ has a unique solution $x_{c}=e^{-\beta_{c}} \in(0,1){ }^{19}$ Then $Z(\beta)$ in (3.5) is well defined for $\beta>\beta_{c}$ and diverges $Z \sim\left(\beta-\beta_{c}\right)^{-1}$ for $\beta \rightarrow \beta_{c} .{ }^{20}$

A correlation between increasing $N$ and the temperature is clear also from the method we used to derive the $N \rightarrow \infty$ limit of the small $x$ expansions of $Z$ in section 3 . As we remarked there, the coefficients of these expansions come from the evaluation of group integrals like (3.6) and these are independent of $N$ as soon as it is larger than some number depending on the degree $\kappa$ in (3.7). As the temperature is increased so that $x \rightarrow 1$, more terms in the small $x$ expansion are needed to accurately describe $Z$. However, at higher orders in $x$, the typical $\kappa$ appearing in the computation increases and one needs to go to larger (but finite) values of $N$ to match the $N=\infty$ limit. This indicates that there is a tension between increasing the temperature and taking the large $N$ limit.

The standard way to systematically describe what happens as the temperature is increased is to consider the distribution of eigenvalues of the group element $U$ in (2.7) that dominates the partition function at certain temperature. At large $N$, the eigenvalue distribution may be approximated by a continuous density $\rho(\alpha)$ with $\alpha \in(-\pi, \pi)$ obeying

$$
\rho(\alpha) \geq 0, \quad \quad \int_{-\pi}^{\pi} d \alpha \rho(\alpha)=1 .
$$

In general, there may be transition points when one passes from a phase where $\rho>0$ is non-zero everywhere on $(-\pi, \pi)$ to a phase where $\rho>0$ only on an interval $\left(-\alpha_{0},-\alpha_{0}\right) \subset$ $(-\pi, \pi)$ (see, e.g., [53]). This is what happens in the vector and adjoint cases $[10,15]$. On general grounds, a transition may be expected when in (2.7) there is balance between the temperature-independent measure term $\sim N^{2}$ and the character-dependent term in the exponent.

As we will see below, this leads to a simple condition

$$
N^{2} \sim N^{p} z_{\Phi}\left(x_{c}\right), \quad x_{c}=e^{-1 / T_{c}},
$$

\footnotetext{
${ }^{19}$ We have always $z_{\Phi}^{\prime}(x)>0, z_{\Phi}(0)=0$. Besides, $z_{\Phi}(1)>1$ if there are at least two single-particle states.

${ }^{20}$ This is consistent with the Hagedorn behaviour $\rho(E) \simeq e^{\beta_{c} E}$ where $\rho(E)$ is the density of states appearing in the the partition function written as $Z=\int d E \rho(E) x^{E}$. The existence of the maximal temperature $T_{c}=1 / \beta_{c}$ is an unphysical feature which is a consequence of the fact that the assumption that the number of relevant states is much smaller than $N^{2}$ fails around $T_{c}$.
} 
where $p=1,2,3, \ldots$ for the vector, adjoint, 3 -plet representation, etc., cases. In the vector case, $x_{c} \rightarrow 1$ as $N \rightarrow \infty$ and taking into account that $z_{\Phi}(x) \stackrel{x \rightarrow 1}{\sim} T^{d-1}$ (cf. (2.3)) we find that

$$
T_{c}^{\text {vector }} \sim N^{\frac{1}{d-1}} \gg 1 .
$$

In the adjoint case, (4.2) gives $T_{c}$ which is independent of $N$,

$$
T_{c}^{\text {adjoint }} \sim 1 .
$$

In the 3-plet case, we are then to expect $T_{c}$ to vanish as $N \rightarrow \infty$ in a way depending on a detailed small $x$ behaviour of $z_{\Phi}(x)$. For example, for a scalar field theory in $d$ dimensions we find from $(2.3)$

$$
T_{c}^{3 \text {-plet }} \sim \frac{d-2}{2} \frac{1}{\log N} \ll 1 .
$$

The scalar contribution is dominant in the $x \rightarrow 0$ limit also when one considers a collection of fields in (2.3), (2.4) like the $(2,0)$ tensor multiplet in $6 \mathrm{~d}$, where thus $T_{c}^{3-\text { plet }} \sim \frac{10}{\log N}$.

The physical reason why $T_{c} \rightarrow 0$ at large $N$ in the case of the 3 -plet representation is that the number of states (operators) grows too quickly with increasing energy (dimension). ${ }^{21}$ Details of this picture will be worked out in the remainder of this section. In particular, in the 3-plet case we will confirm that the phase structure depends on the value of $N z_{\Phi}(x)$ as implied by (4.2).

At large $N$, we shall find a first order discontinuous transition between a phase (where $N z_{\Phi}$ is smaller than a critical value $\left.\left(N z_{\Phi}\right)_{c}\right)$ with $\rho>0$ everywhere on $(-\pi, \pi)$ and a phase (where $N z_{\Phi}>\left(N z_{\Phi}\right)_{c}$ ) with $\rho>0$ only for $|\alpha| \leq \alpha_{0}$ with $\alpha_{0} \sim\left(N z_{\Phi}\right)^{-1 / 2}$. We shall find that the transition point corresponds to $\left(N z_{\Phi}\right)_{c}=\frac{9}{16}$. At any temperature, for a sufficiently large $N$ the system will be in the second phase. This means that at large $N$ the critical temperature is approaching zero.

\subsection{Large $N$ limit in terms of eigenvalue density}

The integration over $U$ in (2.7) may be represented in terms of the eigenvalues of the unitary matrix $\left\{e^{i \alpha_{i}}\right\}$ with $-\pi<\alpha_{i} \leq \pi$

$$
\int d U=\prod_{i=1}^{N} \int_{-\pi}^{\pi} d \alpha_{i} \prod_{i<j} \sin ^{2} \frac{\alpha_{i}-\alpha_{j}}{2}, \quad \operatorname{tr}(U)=\sum_{i} e^{i \alpha_{i}}
$$

Using the explicit form of the characters in (2.9), we get

$$
\begin{aligned}
Z & =\int d \boldsymbol{\alpha} e^{-S(\boldsymbol{\alpha}, x)} \\
S(\boldsymbol{\alpha}, x) & =-\frac{1}{2} \sum_{i \neq j} \log \sin ^{2} \frac{\alpha_{i}-\alpha_{j}}{2}+\sum_{m=1}^{\infty} c_{m}(x) \mathcal{V}(m \boldsymbol{\alpha}), \quad c_{m} \equiv-\frac{1}{m} z_{\Phi}\left(x^{m}\right),
\end{aligned}
$$

\footnotetext{
${ }^{21} \mathrm{~A}$ possible analogy is with a $1 \mathrm{~d}$ Ising model which has $T_{c}=0$ when the length $N$ of the spin chain is infinite. There the aligned "all spins up" (or "all spins down") configuration $\uparrow \uparrow \ldots \uparrow$ has a finite energy gap compared to each of the configurations of the form $\uparrow \uparrow \ldots \uparrow \downarrow \downarrow \ldots \downarrow$. Thus, for large $N$, the variation of the free energy $\Delta F=\Delta \mathcal{E}-T \Delta S \sim-T \log N$ is negative and the entropy term destabilises the ordered configuration for any $T$.
} 
where

$$
\begin{aligned}
\mathcal{V}^{\text {vector }}(\boldsymbol{\alpha}) & =2 \sum_{i=1}^{N} \cos \alpha_{i}, \\
\mathcal{V}^{\text {adjoint }}(\boldsymbol{\alpha}) & =\sum_{i, j=1}^{N} \cos \left(\alpha_{i}-\alpha_{j}\right), \\
\mathcal{V}^{3 \text {-plet }}(\boldsymbol{\alpha}) & =2 \sum_{i, j, k=1}^{N} \cos \left(\alpha_{i}+\alpha_{j}+\alpha_{k}\right) .
\end{aligned}
$$

The extremum condition for $S(\boldsymbol{\alpha}, x)$ in (4.8) is, e.g., in the 3-plet case

$$
\sum_{j \neq i} \cot \frac{\alpha_{i}-\alpha_{j}}{2}-6 \sum_{m=1}^{\infty} z_{\Phi}\left(x^{m}\right) \sum_{j, k=1}^{N} \sin \left[m\left(\alpha_{i}+\alpha_{j}+\alpha_{k}\right)\right]=0 .
$$

Let us follow $[10,12,15]$ and replace the integration over $\boldsymbol{\alpha}$ by the integration over the eigenvalue density $\rho(\alpha)$ which is a periodic function on the unit circle $\alpha \in(-\pi, \pi)^{22}$

$$
\rho(\alpha)=\frac{1}{N} \sum_{n=1}^{N} \delta\left(\alpha-\alpha_{i}\right) .
$$

It satisfies the conditions (4.1) by construction. Then the action in (4.8) becomes

$$
S(\rho, x)=S_{M}(\rho)+V(\rho, x),
$$

with the measure term

$$
\begin{aligned}
S_{M} & =N^{2} \int d \alpha d \alpha^{\prime} K\left(\alpha-\alpha^{\prime}\right) \rho(\alpha) \rho\left(\alpha^{\prime}\right), \\
K(\alpha) & =-\frac{1}{2} \log (2-2 \cos \alpha)=\sum_{m=1}^{\infty} \frac{1}{m} \cos (m \alpha),
\end{aligned}
$$

and the potential term

$$
\begin{aligned}
V^{\text {vector }} & =2 N \int d \alpha \rho(\alpha) \sum_{m=1}^{\infty} c_{m}(x) \cos (m \alpha), \\
V^{\text {adjoint }} & =N^{2} \int d \alpha d \alpha^{\prime} \rho(\alpha) \rho\left(\alpha^{\prime}\right) \sum_{m=1}^{\infty} c_{m}(x) \cos \left[m\left(\alpha-\alpha^{\prime}\right)\right], \\
V^{3 \text {-plet }} & =2 N^{3} \int d \alpha d \alpha^{\prime} d \alpha^{\prime \prime} \rho(\alpha) \rho\left(\alpha^{\prime}\right) \rho\left(\alpha^{\prime \prime}\right) \sum_{m=1}^{\infty} c_{m}(x) \cos \left[m\left(\alpha+\alpha^{\prime}+\alpha^{\prime \prime}\right)\right] .
\end{aligned}
$$

The integral expression for $Z$ in (4.7) can then be written as a path integral over the field $\rho(\alpha)$ subject to the constraints (4.1) with the action (4.14).

As the constant part of $\rho(\alpha)$ drops out of (4.15)-(4.19), the $\rho(\alpha)=\frac{1}{2 \pi}$ is always a stationary point of the action (4.14). Let us first briefly review the vector and adjoint cases where a perturbative expansion around this constant density is sensible and then turn to the 3-plet case where this is not the case.

\footnotetext{
${ }^{22}$ Here the Dirac delta function is the periodic one: $\delta(\alpha) \rightarrow \sum_{k \in \mathbb{Z}} \delta(\alpha+2 \pi k)$.
} 


\subsection{Vector and adjoint cases}

One may expand $\rho(\alpha)$ in Fourier modes as

$$
\rho(\alpha)=\frac{1}{2 \pi}+\frac{1}{N}\left[\frac{1}{\pi} \sum_{m=1}^{\infty} \rho_{m}^{+} \cos (m \alpha)+\frac{1}{\pi} \sum_{m=1}^{\infty} \rho_{m}^{-} \sin (m \alpha)\right] .
$$

This expansion is meaningful if the $\left\{\rho_{m}^{ \pm}\right\}$variables turn out to have a some stationary point with bounded fluctuations. Then, at large $N$, the positivity constraint on $\rho$ in (4.1) will not be violated. Written in terms of the variables $\left\{\rho_{m}\right\}$ the measure term (4.15) becomes

$$
S_{M}=\sum_{m=1}^{\infty} \frac{1}{m}\left[\left(\rho_{m}^{+}\right)^{2}+\left(\rho_{m}^{-}\right)^{2}\right]
$$

while the potentials in (4.17) and (4.18) take the form

$$
V^{\text {vector }}=2 \sum_{m=1}^{\infty} c_{m} \rho_{m}^{+}, \quad V^{\text {adjoint }}=\sum_{m=1}^{\infty} c_{m}\left[\left(\rho_{m}^{+}\right)^{2}+\left(\rho_{m}^{-}\right)^{2}\right] .
$$

In the vector case, the resulting action (4.14) is stationary at

$$
\rho_{m}^{+}=-m c_{m}=z_{\Phi}\left(x^{m}\right), \quad \rho_{m}^{-}=0,
$$

and, as a result, we find the same expression for $Z$ as given earlier in (3.3)

$$
\log Z^{\text {vector }}=\sum_{m=1}^{\infty} \frac{1}{m}\left[z_{\Phi}\left(x^{m}\right)\right]^{2} .
$$

The same expression is found by doing the Gaussian integral over $\rho_{m}^{ \pm}$in (4.7).

In the adjoint case the total action is

$$
S^{\text {adjoint }}=\sum_{m=1}^{\infty} \frac{1+m c_{m}}{m}\left[\left(\rho_{m}^{+}\right)^{2}+\left(\rho_{m}^{-}\right)^{2}\right]=\sum_{m=1}^{\infty} \frac{1-z_{\Phi}\left(x^{m}\right)}{m}\left[\left(\rho_{m}^{+}\right)^{2}+\left(\rho_{m}^{-}\right)^{2}\right] .
$$

Integrating over $\rho_{m}^{ \pm}$in (4.7) with the normalization $Z(x=0)=1$, we then obtain

$$
Z^{\text {adjoint }}=\prod_{m=1}^{\infty}\left[1-z_{\Phi}\left(x^{m}\right)\right]^{-1} \rightarrow \log Z^{\text {adjoint }}=-\sum_{m=1}^{\infty} \log \left[1-z_{\Phi}\left(x^{m}\right)\right]
$$

which is the same as (3.5) quoted earlier.

A similar derivation of $Z$ applies in the case when the adjoint representation is replaced by 2-plet one $N \otimes N$ or its (anti) symmetric version (see appendix A).

As discussed in section 4.1, if the temperature (and thus $x$ and $z_{\Phi}(x)$ ) are small enough, the expressions in (4.24) and (4.26) converge and the expansion (4.20) is well behaved and describes a strictly positive density. Increasing the temperature we reach a transition point where $\rho$ starts to be zero only on a subset $\left(-\alpha_{0}, \alpha_{0}\right) \subset(-\pi, \pi)$. In the vector case, this transition happens at $T_{c} \sim N^{\frac{1}{d-1}}$ as in (4.3). The expansion (4.20) is no longer valid above this critical temperature. In adjoint case, the critical temperature is determined by the 
the condition $z_{\Phi}(x)=1$ when the fluctuations of modes in (4.25) are no longer suppressed. Thus in this case we get the critical temperature $T_{c} \sim 1$ in (4.4) which is independent of $N$. Details of the form of $\rho(\alpha)$ in the two phases for the vector and adjoint models may be found in $[10,15,19]$.

In the low temperature phase where the expansion (4.20) is valid, the value of the action (4.14) (i.e. the measure term (4.21) plus potential in (4.22)) and thus the resulting large $N$ partition function (4.24) or (4.26) does not depend on $N$, i.e. $\log Z=\mathcal{O}\left(N^{0}\right)$.

The stationary point of the action (4.14) is given by the analog of eq. (4.8) written in terms of $\rho(\alpha)$ (cf. (4.28) below). Assuming it admits a non-trivial solution for $\rho(\alpha)$ (due to a balance between the measure and potential contributions to the stationary point equation) then in the higher temperature $T>T_{c}$ phase the resulting value of the action (4.14) at the stationary point will scale as the measure term, i.e. as $N^{2}$. Thus the stationarypoint approximation will be valid in the large $N$ limit with $\log Z=\mathcal{O}\left(N^{2}\right)$ in the hightemperature phase (see (C.6), (C.11)).

In the 3-plet case discussed below the low temperature phase will be shrinking with increasing $N$ (with $T_{c} \rightarrow 0$ ) while the action and $\log Z$ will be scaling again as $N^{2}$ at the non-trivial stationary-point solution of (4.28).

\subsection{3 -plet case}

In the 3-plet case, using (4.20) in the potential term (4.19) we get

$$
V^{3 \text {-plet }}=2 \sum_{m=1}^{\infty} c_{m}\left[\left(\rho_{m}^{+}\right)^{3}-3 \rho_{m}^{+}\left(\rho_{m}^{-}\right)^{2}\right] .
$$

The resulting action given by the sum of (4.21) and (4.27) is unbounded from below (in particular, the analog of (4.7) giving $Z$ as an integral over $\rho_{m}^{ \pm}$does not converge). Thus in the 3 -plet case the constant density $\rho=\frac{1}{2 \pi}$ is really a saddle and not a minimum even at low temperatures; the Fourier coefficients $\rho_{m}^{ \pm}$in (4.20) tend to be large and this violates the fundamental positivity condition on $\rho(\alpha)$ in (4.1).

A phase transition from the trivial homogeneous phase $\rho=\frac{1}{2 \pi}$ should happen when the cubic in $\rho$ term in the action (4.14), (4.19) becomes of order of the quadratic measure one in (4.15). In the vector case the transition point is when we balance a measure term (4.15) $\sim N^{2}$ against the potential term $(4.17) \sim N z_{\Phi}(x)$. In the adjoint case, both terms (4.15) and (4.18) are of the same order $\sim N^{2}$. In the 3-plet case the potential term (4.19) scales as $N^{3} z_{\Phi}(x)$ and we are led to the condition in (4.2) (in a sense, the vector and the 3-plet cases are opposite to each other with the adjoint case being in between).

Below we will explore the 3-plet case in more detail, by first studying numerically the eigenvalue distribution and then presenting some analytical expressions.

\subsubsection{Numerical analysis}

As a preliminary step, we can try to determine numerically the solution to the extremum condition (4.12). We shall sum over $m$ in (4.12) with $1 \leq m \leq M$ to investigate the effect of higher harmonics. We shall consider the case of $4 \mathrm{~d}$ scalar with $z_{\Phi}(x)=\frac{x(1+x)}{(1-x)^{3}}($ see $(2.3))$. Solving numerically (4.12) with $N=40, M=4$ we find the following behaviour: 

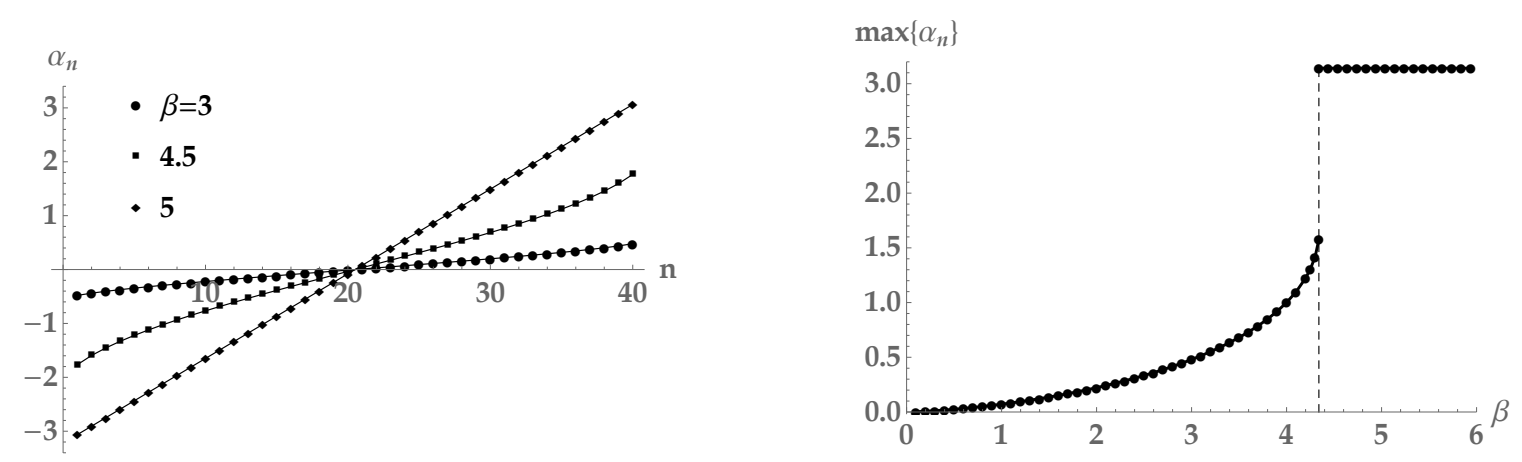

Figure 1. Exact eigenvalues for $N=40, M=4$ and three values of $\beta$ well below, just below and just above the transition. The right graph represents the plot of the maximal eigenvalue that demonstrates that it jumps at a certain temperature from a value $\simeq \frac{\pi}{2}$ to $\pi$.

1. At high temperature, the eigenvalues $\alpha_{i}$ are distributed on $\left(-\alpha_{0}, \alpha_{0}\right) \subset(-\pi, \pi)$ with $\alpha_{0} \rightarrow 0$ as $\beta \rightarrow 0$. Their density is approximately flat near the center of the interval, but with deviations at the edges.

2. As the temperature is reduced, there is a transition point where the distribution becomes essentially uniform over the whole interval $(-\pi, \pi)$.

This is illustrated in figure 1 where we show in the left graph the exact eigenvalues at $\beta=3,4.5,5$ that are well below, just below and above the transition. The right graph shows $\max \left|\alpha_{n}\right|$ as a function of $\beta$ to emphasize the rapid jump from a value $\simeq \frac{\pi}{2}$ to $\pi$ at a critical temperature. In appendix C.1 we will consider explicitly the small $N=2$ case where these features are already visible.

\subsubsection{Exact solution for the eigenvalue density at large $N$}

The features observed in the numerical analysis may be explained analytically as follows. Let us return to the stationary point condition in eq. (4.12) expressing it in terms of $\rho(\alpha)$ :

$$
\int d \alpha^{\prime} \rho\left(\alpha^{\prime}\right) \cot \frac{\alpha-\alpha^{\prime}}{2}=6 N \sum_{m=1}^{\infty} z_{\Phi}\left(x^{m}\right) \int d \alpha^{\prime} d \alpha^{\prime \prime} \rho\left(\alpha^{\prime}\right) \rho\left(\alpha^{\prime \prime}\right) \sin \left[m\left(\alpha+\alpha^{\prime}+\alpha^{\prime \prime}\right)\right]
$$

Let us assume that $\rho$ is symmetric and supported on $\left(-\alpha_{0}, \alpha_{0}\right)$ and thus write (4.28) as

$$
\begin{aligned}
\int d \alpha^{\prime} \rho\left(\alpha^{\prime}\right) \cot \frac{\alpha-\alpha^{\prime}}{2} & =6 N \sum_{m=1}^{\infty} z_{\Phi}\left(x^{m}\right) \int d \alpha^{\prime} d \alpha^{\prime \prime} \rho\left(\alpha^{\prime}\right) \rho\left(\alpha^{\prime \prime}\right) \sin (m \alpha) \cos \left[m\left(\alpha^{\prime}+\alpha^{\prime \prime}\right)\right] \\
& =2 \sum_{m=1}^{\infty} a_{m} \rho_{m}^{2} \sin (m \alpha)
\end{aligned}
$$

where

$$
a_{m}=3 N z_{\Phi}\left(x^{m}\right), \quad \rho_{m}=\int d \alpha \rho(\alpha) \cos (m \alpha)
$$


Eq. (4.29) is same as eq. (5.20) of [15] and thus its solution may be written as

$$
\begin{aligned}
\rho(\alpha) & =\frac{1}{\pi} \sqrt{\sin ^{2} \frac{\alpha_{0}}{2}-\sin ^{2} \frac{\alpha}{2}} \sum_{k=1}^{\infty} Q_{k} \cos \left[\left(k-\frac{1}{2}\right) \alpha\right], \\
Q_{k} & =2 \sum_{\ell=0}^{\infty} a_{k+\ell} \rho_{k+\ell}^{2} P_{\ell}\left(\cos \alpha_{0}\right),
\end{aligned}
$$

where $P_{\ell}$ is the Legendre polynomial. To simplify the presentation, let us first consider a model with just one harmonic $\rho_{1}$ present in the r.h.s. of (4.29) which should be a good approximation for large $\beta$ when $x=e^{-\beta} \ll 1$ and thus the value of $a_{m}$ in (4.30) decreases with $m$. Then

$$
\rho(\alpha)=\frac{2}{\pi} a_{1} \rho_{1}^{2} \sqrt{\sin ^{2} \frac{\alpha_{0}}{2}-\sin ^{2} \frac{\alpha}{2}} \cos \frac{\alpha}{2} .
$$

We still need to impose the self-consistency equations

$$
\int_{-\alpha_{0}}^{\alpha_{0}} d \alpha \rho(\alpha)=1, \quad \int_{-\alpha_{0}}^{\alpha_{0}} d \alpha \rho(\alpha) \cos \alpha=\rho_{1} .
$$

We then find

$$
\begin{aligned}
2 a_{1} \rho_{1}^{2} u & =1, & 2 a_{1} \rho_{1}^{2} u\left(1-\frac{1}{2} u\right) & =\rho_{1}, \quad u \equiv \sin ^{2} \frac{\alpha_{0}}{2} \in(0,1) \\
1-\frac{1}{2} u & =\left(2 a_{1} u\right)^{-1 / 2}, & a_{1} & =3 N z_{\Phi}\left(e^{-\beta}\right) .
\end{aligned}
$$

Thus $u$ solves a cubic equation. For large $a_{1}$ we get a consistent solution

$$
u=\frac{1}{2 a_{1}}+\frac{1}{4 a_{1}^{2}}+\ldots \rightarrow \alpha_{0}=\left[\frac{3}{2} N z_{\Phi}\left(e^{-\beta}\right)\right]^{-1 / 2}+\ldots
$$

As $a_{1}$ decreases, we find a solution with $u \in(0,1)$ only up to the point

$$
a_{1}=\frac{27}{16}, \quad \text { i.e. } \quad u=\frac{2}{3} .
$$

This limiting value corresponds to the maximal width of the interval being

$$
\alpha_{0}=2 \arctan \sqrt{2} \simeq 0.6 \pi
$$

To summarize, including just one harmonic in the sum in (4.29), for each temperature and $N$ such that

$$
N z_{\Phi}\left(e^{-\beta}\right)>\frac{9}{16}
$$

we get the eigenvalue distribution

$$
\rho(\alpha)= \begin{cases}\frac{1}{\pi \sin ^{2} \frac{\alpha_{0}}{2}} \sqrt{u-\sin ^{2} \frac{\alpha}{2}} \cos \frac{\alpha}{2}, & |\alpha|<\alpha_{0} \\ 0 & \alpha_{0}<|\alpha| \leq \pi\end{cases}
$$




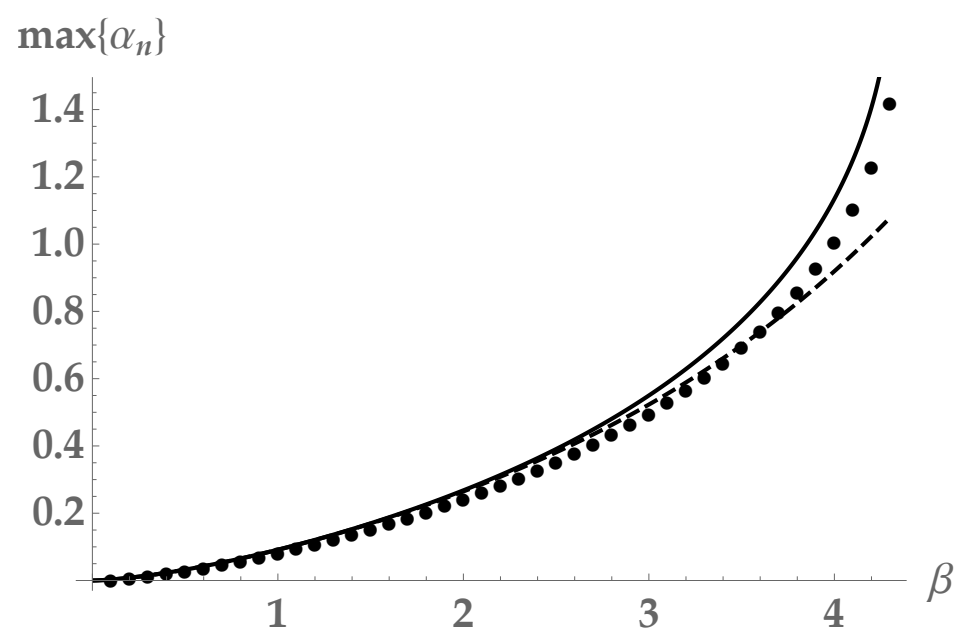

Figure 2. Comparison between the edge of the exact eigenvalue distribution at $N=40$ in the case of one harmonic in the potential term in (4.12) (solid circles) with the solution of (4.42) (solid line). We also show the high temperature approximation (4.46) in the one-harmonic case (dashed line).

where $u=\sin ^{2} \frac{\alpha_{0}}{2}$ is determined by the relation (4.36), i.e.

$$
\frac{3}{2} u(2-u)^{2}=\left[N z_{\Phi}\left(e^{-\beta}\right)\right]^{-1}
$$

For $\frac{9}{16}<N z_{\Phi}\left(e^{-\beta}\right)<\frac{2}{3}$ there are two solutions $u_{1}, u_{2}$ with $0<u_{1}<u_{2}<1$. Here $u_{1}$ is a minimum of the action, while $u_{2}$ is a local maximum, see appendix C.2. A numerical test of (4.42) is shown in figure 2 where we compare its prediction with the edge of the exact eigenvalue distribution at $N=40$ found by taking just one harmonic in the potential term in (4.8).

Including up to $M$ higher harmonics, the equations (4.35) are replaced by similar ones involving $u$ and $\rho_{1}, \ldots, \rho_{M}$. The solution depends on $N$ and $\beta$ through the combinations $a_{m}=3 N z_{\Phi}\left(e^{-m \beta}\right), \quad m=1, \ldots, M$ (see (4.29), (4.30)). For large $N$, the critical inverse temperature $\beta_{c}(N, M)$ admits a finite limit that may, in principle, depend on $M$. In fact, $a_{c}$ defined as

$$
a_{c}(M) \equiv \lim _{N \rightarrow \infty} 3 N z_{\Phi}\left(e^{-\beta_{c}(N, M)}\right) .
$$

is independent of $M$. To see why, consider, for example, $z_{\Phi}(x)=\frac{x(1+x)}{(1-x)^{3}}$ corresponding to $4 \mathrm{~d}$ scalar theory. If we solve $a_{c}=3 N z_{\Phi}\left(e^{-\beta_{c}}\right)$ for $\beta_{c}$ at large $N$, we get $\beta_{c}=$ $-\log \frac{a_{c}}{3 N}+\frac{4 a_{c}}{3 N}-\frac{5 a_{c}^{2}}{3 N^{2}}+\ldots$ The coupling parameters $a_{m}=3 N z_{\Phi}\left(e^{-m \beta}\right)$ for higher harmonics in $(4.29),(4.30)$ are then subleading, e.g., $3 N z_{\Phi}\left(e^{-2 \beta_{c}}\right)=\frac{a_{c}^{2}}{3 N}-\frac{8 a_{c}^{3}}{9 N^{2}}+\ldots$, and in general $3 N z_{\Phi}\left(e^{-m \beta_{c}}\right)=\frac{a_{c}^{m}}{(3 N)^{m-1}}+\ldots$ Thus,

$$
a_{c}=a_{c}(M=1)=\frac{27}{16}
$$

which is the value of $a_{1}$ found in (4.38). 
To give an example, with $M=3$ harmonics and taking $N=50,60, \ldots, 100$ we get

\begin{tabular}{|ccccccc|}
\hline$N$ & 50 & 60 & 70 & 80 & 90 & 100 \\
\hline$N z_{\Phi}\left(e^{\beta_{c}(N, M=3)}\right)$ & 0.562392 & 0.562409 & 0.562421 & 0.56243 & 0.562438 & 0.562444 \\
\hline
\end{tabular}

A quadratic fit with $1 / N$ and $1 / N^{2}$ corrections gives $a_{c}(M=3)=3 \times 0.562502$ which is equal to $\frac{27}{16}$ in $(4.44)$ with a $10^{-6}$ relative precision.

As we will show in appendix C.3, deep into the high temperature phase, the 1-harmonic result (4.37) is replaced by

$$
\alpha_{0}=\left[\frac{3}{2} N \sum_{m=1}^{\infty} m z_{\Phi}\left(e^{-m \beta}\right)\right]^{-1 / 2}+\ldots
$$

Similar results are found in the case when the general 3-plet representation is replaced by the symmetric or antisymmetric one: as we will show in appendix C.4, the large $N$ behaviour is the same in all of these cases.

\section{Concluding remarks}

In this paper we discussed singlet partition function $Z$ of conformal theories defined by free fields in higher representations of an internal symmetry group. We observed that starting with rank 3 tensor case the number of singlet states grows so fast with the energy that the small temperature expansion of $Z$ has zero radius of convergence in the $N=\infty$ limit. This is reflected in the vanishing of the critical temperature $T_{c}$ at $N=\infty$. For large but finite $N$ there are two phases: $T<T_{c}$ and $T>T_{c}$, with $\log Z \sim N^{2}$ in the higher temperature phase (same scaling as found in the vector and adjoint representation cases).

We have concentrated on the case of the $p$-fundamental representation of $\mathrm{U}(N)$ but similar conclusions are true also for the $[\mathrm{U}(N)]^{p}$ invariant singlet partition function of $p$ tensors with inequivalent indices (see appendix F). The same qualitative behaviour is found also when $\mathrm{U}(N)$ symmetry is replaced by $O(N)$ (cf. appendix D).

One open question is about possible implications for the AdS dual of the free $p$-plet or $p$-tensor CFT. The rich spectrum of singlet operators implies the presence of infinite sequences of massive fields in AdS (suggestive of a "tensionless membrane" spectrum in the $p=3$ case). It would be interesting to shed further light on this by studying simplest correlation functions of operators like $\bar{\phi}_{i j k} \phi_{i j k}$ (dual to a scalar in AdS) by generalizing the discussion of the vector and adjoint cases in [19].

For the fields in the vector and adjoint representations the large $N$ free energy or 1-loop $\log Z$ of all higher spin fields in thermal AdS scales as $\mathcal{O}\left(N^{0}\right)$ and matches the corresponding boundary CFT free energy in the low-temperature phase in (3.3), (3.5). In the high temperature phase the boundary CFT free energy is $\mathcal{O}\left(N^{2}\right)$; that formally agrees with an AdS black-hole scaling in the adjoint case $[54,55]$. In the vectorial case where $T_{c}$ grows with $N$ and thus the high temperature phase is not obviously attainable, a possibility of similar matching remains an open question [10] (the classical AdS action here scales as $N$ and thus a classical thermal object would contribute $\mathcal{O}(N)$ to the free energy). 
In the 3-plet case the 1-loop partition function in thermal AdS computed for the full spectrum of fields dual to singlet conformal operators in the large $N$ limit should also be expected to be given by an asymptotic series matching the low temperature phase expression for $\log Z(x)=\mathcal{O}\left(N^{0}\right)$ in (3.32). The high temperature phase result $\log Z(x)=$ $\mathcal{O}\left(N^{2}\right)$ here appears to be subleading to any potential contribution coming from a classical AdS action as that should scale as $\mathcal{O}\left(N^{3}\right)$ (the coefficient in front of the AdS action should be $N^{3}$ to match the correlation functions in the free 3-plet CFT [27]).

Another important question is how these conclusions may change in an interacting CFT, e.g., whether $T_{c}$ may become finite at a non-trivial large $N$ fixed point. This is of particular interest in the case of the $(2,0)$ tensor multiplet theory in $6 \mathrm{~d}$ that should have an $\mathrm{AdS}_{7}$ dual with a supergravity limit in the $N \rightarrow \infty$ limit admitting black holes and thus predicting $N^{3}$ scaling of the free energy [20, 25].

\section{Acknowledgments}

We are grateful to I. Klebanov and G. Tarnopolsky for very useful discussions and also thank E. Joung for comments. The work of AAT was supported by the ERC Advanced grant no. 290456, the STFC Consolidated grant ST/L00044X/1 and the Russian Science Foundation grant 14-42-00047 at Lebedev Institute.

\section{A $\quad N=\infty$ partition function for 2-plet representation of $\mathrm{U}(N)$}

In addition to the adjoint representation $N \otimes \bar{N}$ one may consider also another rank 2 tensor representation $-N \otimes N$ or 2-plet of $\mathrm{U}(N)$. The corresponding real representation in (2.7) is $R=N \otimes N+\bar{N} \otimes \bar{N}$ and thus $\chi_{R}(U)=[\operatorname{tr}(U)]^{2}+\left[\operatorname{tr}\left(U^{-1}\right)\right]^{2}$ (see (2.10)). The resulting potential in (4.8) is then (cf. (4.9)-(4.11))

$$
V^{2-\operatorname{plet}}(\rho)=2 N^{2} \int d \alpha d \alpha^{\prime} \rho(\alpha) \rho\left(\alpha^{\prime}\right) \sum_{m=1}^{\infty} c_{m} \cos \left(m\left(\alpha+\alpha^{\prime}\right)\right) .
$$

In terms of the Fourier coefficients $\rho_{m}^{ \pm}$in (4.20) we get

$$
V^{2 \text {-plet }}=2 \sum_{m=1}^{\infty} c_{m}\left[\left(\rho_{m}^{+}\right)^{2}-\left(\rho_{m}^{-}\right)^{2}\right]
$$

As in the adjoint case $(4.25),(4.26)$, integrating over $\rho_{m}^{ \pm}$we get for the $N=\infty$ partition function

$$
\log Z^{2 \text {-plet }}=-\frac{1}{2} \sum_{m=1}^{\infty} \log \left(1-\left[2 z_{\Phi}\left(x^{m}\right)\right]^{2}\right),
$$

in agreement with (3.29). The corresponding single-trace partition function is given in (E.8). The expression (A.3) is valid in the low temperature phase, i.e. for temperatures below the critical one where $2 z_{\Phi}\left(x_{c}\right) \sim 1$.

For the symmetric 2-plet representation where $R=(N \otimes N)_{\text {sym }}+(\bar{N} \otimes \bar{N})_{\text {sym }}$ and

$$
\chi_{R}(U)=\frac{1}{2}[\operatorname{tr}(U)]^{2}+\frac{1}{2} \operatorname{tr}\left(U^{2}\right),
$$


we get instead of (A.1)

$$
\begin{aligned}
V^{2 \text {-plet }}+ & =N^{2} \int d \alpha d \alpha^{\prime} \rho(\alpha) \rho\left(\alpha^{\prime}\right) \sum_{m=1}^{\infty} c_{m} \cos \left(m\left(\alpha+\alpha^{\prime}\right)\right)+N \int d \alpha \rho(\alpha) \sum_{m=1}^{\infty} c_{m} \cos (2 m \alpha) \\
& =\sum_{m=1}^{\infty} c_{m}\left[\left(\rho_{m}^{+}\right)^{2}-\left(\rho_{m}^{-}\right)^{2}\right]+\sum_{m=1}^{\infty} c_{m} \rho_{2 m}^{+} .
\end{aligned}
$$

Adding this to (4.21) and performing again the Gaussian integration over $\rho^{ \pm}$gives (cf. (4.26), (A.3))

$$
\log Z^{2-\text { plet }^{+}}=-\frac{1}{2} \sum_{m=1}^{\infty} \log \left(1-\left[z_{\Phi}\left(x^{m}\right)\right]^{2}\right)+\frac{1}{2} \sum_{m=1}^{\infty} \frac{1}{m} \frac{\left[z_{\Phi}\left(x^{m}\right)\right]^{2}}{1-z_{\Phi}\left(x^{2 m}\right)} .
$$

In the antisymmetric 2-plet representation case there is a relative minus sign in (A.4) and thus in the last term in (A.5) but the final result for $Z$ is again the same as (A.6).

\section{B Finite $N$ low temperature expansion of 3-plet partition function}

Here we will supplement the large $N$ analysis in section 3 with a discussion of the finite $N$ case. At finite $N$, the low temperature expansion of the partition function may still be done by direct expanding (2.7). However, simple expressions like (3.8) or factorization leading to (3.24) are no longer valid. Instead, the group integrals (3.6) must be computed on a case by case basis.

In particular, for a $4 \mathrm{~d}$ scalar in 3 -plet representation, one can compute the following first five terms in the small $X$ expansion of $Z_{\mathrm{S}, 4}^{3 \text {-plet }}$ for the increasing $N$ (the coefficients that are stable under the increase of $N$ are in bold face)

$$
\begin{array}{|lc|}
\hline N & Z_{\mathrm{S}, 4}^{3 \text {-plet }} \\
\hline 2 & 1+5 x^{2}+40 x^{3}+212 x^{4}+1080 x^{5}+6054 x^{6}+\cdots \\
3 & 1+\mathbf{6} x^{2}+\mathbf{4 8} x^{3}+342 x^{4}+2688 x^{5}+21408 x^{6}+\cdots \\
4 & 1+\mathbf{6} x^{2}+\mathbf{4 8} x^{3}+387 x^{4}+3384 x^{5}+31765 x^{6}+\cdots \\
5 & 1+\mathbf{6} x^{2}+\mathbf{4 8} x^{3}+\mathbf{3 9 6} x^{4}+\mathbf{3 5 0 4} x^{5}+35012 x^{6}+\cdots \\
6 & 1+\mathbf{6} x^{2}+\mathbf{4 8} x^{3}+\mathbf{3 9 6} x^{4}+\mathbf{3 5 0 4} x^{5}+35535 x^{6}+\cdots \\
7 & 1+\mathbf{6} x^{2}+\mathbf{4 8} x^{3}+\mathbf{3 9 6} x^{4}+\mathbf{3 5 0 4} x^{5}+\mathbf{3 5 5 8 0} x^{6}+\cdots \\
\hline
\end{array}
$$

The expansions in (B.1) may be derived using the character expansion method that is quite convenient at relatively small $N$ (see, e.g., [49]). Irreducible representations of $\mathrm{U}(N)$ may be labeled by $N$ integers $\boldsymbol{n}=\left(n_{1}, n_{2}, \ldots, n_{N}\right)$ with $n_{1} \geq n_{2} \geq \cdots \geq n_{N} \geq 0$. Denoting by $t_{1}, \ldots, t_{N}$ the eigenvalues of the group element $U$ in the fundamental representation, we obtain the character $\chi_{n}$ from the Weyl formula ( $i, j$ are row and column indices)

$$
\chi_{\boldsymbol{n}}(U)=\frac{\operatorname{det}\left(t_{i}^{n_{j}+N-j}\right)}{\operatorname{det}\left(t_{j}^{N-i}\right)} .
$$


This is a polynomial in the eigenvalues $t_{i}$ with total degree equal to $\sum_{i} n_{i}$. Any polynomial built out of powers of traces $\operatorname{tr}\left(U^{k}\right)$ may be expanded as a finite sum of such characters. Then the group integrals in (3.6) are easily evaluated by exploiting the orthonormality of the characters $\int d U \chi_{\boldsymbol{n}}(U) \overline{\chi_{\boldsymbol{n}^{\prime}}(U)}=\delta_{\boldsymbol{n}, \boldsymbol{n}^{\prime}}$. The dependence on $N$ of the final result is a consequence of the fact that the fine details of the character decomposition also depend on $N$. To give a simple nontrivial example, let us consider the integral

$$
I_{N}=\int d U\left|\left(\operatorname{tr} U^{2}\right)^{2}\right|^{2}, \quad U \in \mathrm{U}(N),
$$

The character expansion of $\left(\operatorname{tr} U^{2}\right)^{2}=\left(\sum_{i=1}^{N} t_{i}^{2}\right)^{2}$ reads

$$
\begin{array}{|ll|}
\hline N & \left(\operatorname{tr} U^{2}\right)^{2} \\
\hline 2 & 2 \chi_{22}-\chi_{31}+\chi_{40} \\
3 & 2 \chi_{220}-\chi_{310}+\chi_{400}-\chi_{211} \\
4 & 2 \chi_{2200}-\chi_{3100}+\chi_{4000}-\chi_{2110}+\chi_{1111} \\
5 & 2 \chi_{22000}-\chi_{31000}+\chi_{40000}-\chi_{21100}+\chi_{11110} \\
6 & 2 \chi_{220000}-\chi_{310000}+\chi_{400000}-\chi_{211000}+\chi_{111100} \\
\hline
\end{array}
$$

and so on. Thus the cases $N=2,3$ are special, but there is a stable pattern for $N \geq 4$. Using the orthogonality of the characters and (B.4) we find for (B.3)

$$
I_{2}=2^{2}+2 \times 1^{2}=6, \quad I_{3}=2^{2}+3 \times 1^{2}=7, \quad I_{N \geq 4}=2^{2}+4 \times 1^{2}=8 .
$$

\section{Details of analysis of large $N$ partition function for 3-plet representa- tion}

\section{C.1 U(2) case}

To compare the large $N$ and the finite $N$ cases, it is useful to consider the lowest non-trivial value of $N=2$. Then the action (4.8) in 3-plet case with just one harmonic included is a function of the single eigenvalue angle $\alpha=\alpha_{1}=-\alpha_{2}$ and inverse temperature $\beta$

$$
S=-\log \sin ^{2} \alpha-16 z_{\Phi}\left(e^{-\beta}\right) \cos ^{3} \alpha .
$$

The left part of figure 3 is the plot of this function for four values of $\beta$. For $\beta<\beta_{c}$ the value of $S$ at the global minimum is negative. This global minimum on the left and the local minimum at $\pi / 2$ on the right become degenerate at $\beta_{c}$. For $\beta>\beta_{c}$ the global minimum is on the right at $\alpha=\pi / 2$.

The transition temperature can be found analytically to be $\beta_{c}=2.454$. The associated eigenvalue is $\alpha_{c}=0.752$. This means that there is a first order (discontinuous) transition. Increasing $\beta$, the eigenvalue $\alpha$ goes from 0 to $\alpha_{c}$ and then jumps to $\alpha=\pi / 2$. Increasing the number of harmonics included in (4.8) does not change this picture qualitatively. This is illustrated in the right plot in figure 3 where we assumed that there are 10 harmonics in (4.8).

What changes at higher $N$ is that the right minimum shifts further to the right, tending to $\alpha=\pi$ for $N \rightarrow \infty$. 


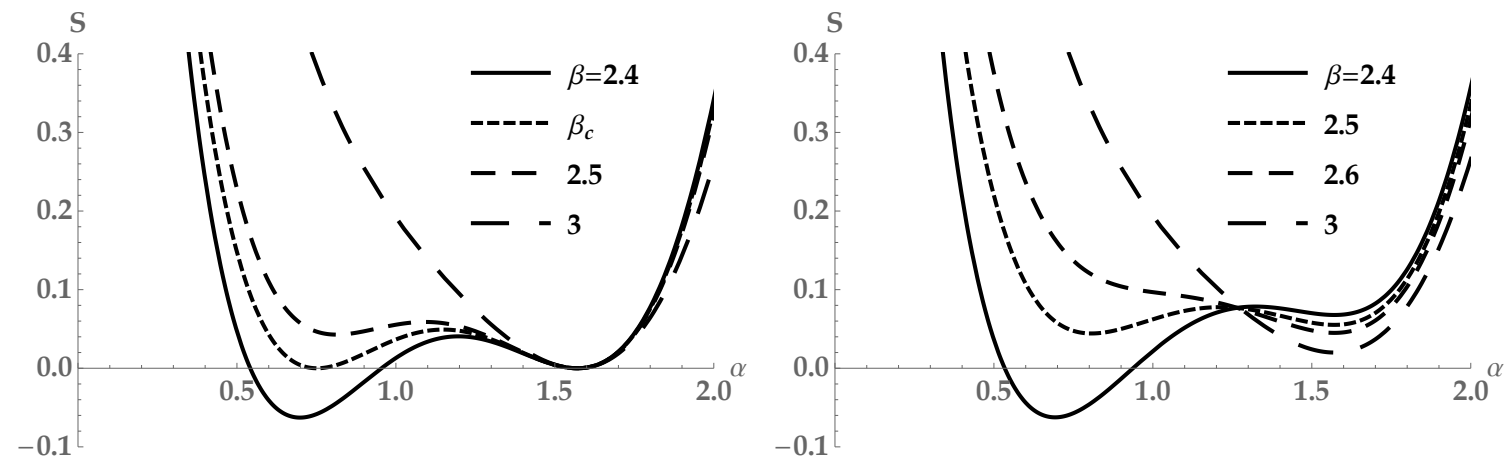

Figure 3. The action $S$ in (4.8) for the $\mathrm{U}(2)$ theory as a function of the eigenvalue $\alpha=\alpha_{1}=-\alpha_{2}$. The left plot is for one and the right plot is for ten harmonics included in (4.8).

\section{C.2 One-harmonic solution: value of the action for the eigenvalue density}

To determine the leading term in the free energy $\log Z$ at the large $N$ saddle point one is to compute the value of the action (4.14) on the solution of (4.28).

Below we shall compute the sum of the two terms in the action (4.14)

$$
\begin{aligned}
S_{M} & =-\frac{1}{2} N^{2} \int d \alpha d \alpha^{\prime} \rho(\alpha) \rho\left(\alpha^{\prime}\right) \log \sin ^{2} \frac{\alpha-\alpha^{\prime}}{2} \\
V & =-2 N^{3} z_{\Phi}\left(e^{-\beta}\right) \int d \alpha d \alpha^{\prime} d \alpha^{\prime \prime} \rho(\alpha) \rho\left(\alpha^{\prime}\right) \rho\left(\alpha^{\prime \prime}\right) \cos \left(\alpha+\alpha^{\prime}+\alpha^{\prime \prime}\right)
\end{aligned}
$$

on the solution (4.33) found in one-harmonic approximation, i.e.

$$
\rho(\alpha)=\frac{1}{u \pi} \sqrt{u-\sin ^{2} \frac{\alpha}{2}} \cos \frac{\alpha}{2}, \quad u=\sin ^{2} \frac{\alpha_{0}}{2} .
$$

Using the identity

$$
-\frac{1}{2} \log \sin ^{2} \frac{\alpha}{2}=\log 2+\sum_{m=1}^{\infty} \frac{1}{m} \cos (m \alpha)
$$

we find for the measure term

$$
S_{M}=-\frac{1}{2} N^{2} \int d \alpha d \alpha^{\prime} \rho(\alpha) \rho\left(\alpha^{\prime}\right) \log \sin ^{2} \frac{\alpha-\alpha^{\prime}}{2}=N^{2}\left(\log 2+\sum_{m=1}^{\infty} \frac{1}{m} \rho_{m}^{2}\right),
$$

where $\rho_{m}$ was defined in (4.30), i.e.

$$
\rho_{m}=\frac{1}{\pi u} \int_{-\alpha_{0}}^{\alpha_{0}} d \alpha \sqrt{u-\sin ^{2} \frac{\alpha}{2}} \cos \frac{\alpha}{2} \cos (m \alpha), \quad u=\sin ^{2} \frac{\alpha_{0}}{2}
$$

Introducing $t=\sin \frac{\alpha}{2} / \sin \frac{\alpha_{0}}{2}$ we get

$$
\rho_{m}=\frac{2}{\pi \sqrt{u}} \int_{0}^{1} d t \sqrt{1-t^{2}} \sqrt{1-u t^{2}} \cos [2 m \arcsin (u t)] .
$$




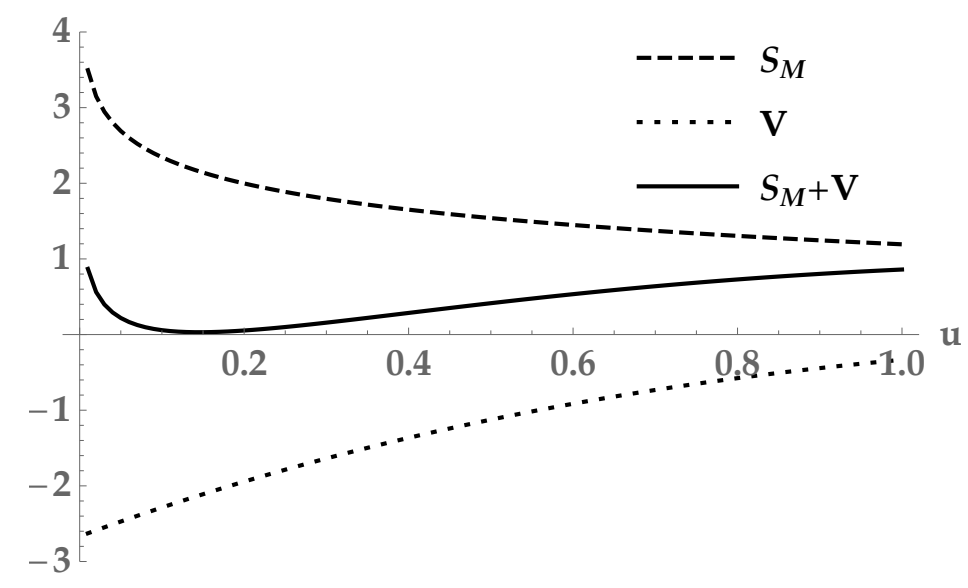

Figure 4. Plot of the measure term $S_{M}$ in (C.6) and the potential term $V$ in (C.12) in the eigenvalue density action at $a_{1}=3 N z_{\Phi}\left(e^{-\beta}\right)=4$. The black line is the total action that has a minimum at the position predicted by (4.36), i.e. at $u \simeq 0.145$.

Using the expansion

$$
\cos [2 m \arcsin (u t)]=\sum_{n=0}^{\infty} \frac{(m)_{n}(-m)_{n}}{(2 n) !}(2 u t)^{2 n},
$$

we obtain from $(\text { C.8 })^{23}$

$$
\rho_{m}=\sum_{n=0}^{m} \frac{(m)_{n}(-m)_{n}}{n !(n+1) !} u^{n}={ }_{2} F_{1}(-m, m, 2 ; u) .
$$

Thus

$$
S_{M}=N^{2}\left(\log 2+\sum_{m=1}^{\infty} \frac{1}{m}\left[{ }_{2} F_{1}(-m, m, 2 ; u)\right]^{2}\right) .
$$

The potential term is given simply by

$$
V=-2 N^{3} z_{\Phi}\left(e^{-\beta}\right) \rho_{1}^{3}=-2 N^{3} z_{\Phi}\left(e^{-\beta}\right)\left(1-\frac{u}{2}\right)^{3} \equiv N^{2} \bar{V}(u),
$$

where we used (4.35), (4.36).

In figure 4 we plot $S_{M}$ and $V$ evaluated as functions of $u$ at $a_{1}=3 N z_{\Phi}\left(e^{-\beta}\right)=4$, i.e. at the value which is above the bound in (4.38). As expected, there is a minimum of the total action $S=S_{M}+V$ located at the value predicted by the cubic equation for $u$ in (4.36).

\section{C.3 Including higher harmonics}

It is easy to generalize the discussion in section 4.4.2 to the case of higher harmonics included in (4.29). Some analytical information may be obtained at least for small $\beta$ where

\footnotetext{
${ }^{23}$ The first cases are $\rho_{0}=1, \quad \rho_{1}=1-\frac{u}{2}, \quad \rho_{2}=1-2 u+u^{2}, \quad \rho_{3}=1-\frac{9 u}{2}+6 u^{2}-\frac{5 u^{3}}{2}, \ldots$ Here $\rho_{0}=1$ is the normalization of $\rho$, while $\rho_{1}$ is consistent with (4.35).
} 
the maximal value of eigenvalues $\alpha_{0}$ is small. We need to solve eq. (4.28), i.e.

$$
\int d \alpha^{\prime} \rho\left(\alpha^{\prime}\right) \cot \frac{\alpha-\alpha^{\prime}}{2}=6 N \sum_{m=1}^{\infty} z_{\Phi}\left(x^{m}\right) \int d \alpha^{\prime} d \alpha^{\prime \prime} \rho\left(\alpha^{\prime}\right) \rho\left(\alpha^{\prime \prime}\right) \sin \left(m\left(\alpha+\alpha^{\prime}+\alpha^{\prime \prime}\right)\right) .
$$

Introducing

$$
\theta=\frac{\alpha}{\alpha_{0}}, \quad \widetilde{\rho}(\theta)=\alpha_{0} \rho\left(\alpha_{0} \theta\right), \quad d \alpha \rho(\alpha)=d \theta \widetilde{\rho}(\theta), \quad \int_{-1}^{1} d \theta \widetilde{\rho}(\theta)=1
$$

and expanding (C.13) in small $\alpha_{0}$, we obtain at the leading order

$$
\int_{-1}^{1} d \theta^{\prime} \frac{\widetilde{\rho}\left(\theta^{\prime}\right)}{\theta-\theta^{\prime}}-3 N \alpha_{0}^{2} \sum_{m=1}^{\infty} m z_{\Phi}\left(e^{-m \beta}\right) \theta=0 .
$$

For a constant parameter $\gamma$, the Hilbert problem

$$
\int_{-1}^{1} d \theta^{\prime} \frac{\widetilde{\rho}\left(\theta^{\prime}\right)}{\theta-\theta^{\prime}}=\gamma \theta, \quad \theta \in(-1,1)
$$

has the unique solution

$$
\widetilde{\rho}(\theta)=\frac{\gamma}{\pi} \sqrt{1-\theta^{2}}
$$

The normalization in (C.14) fixes $\gamma=2$. Comparing with (C.15), we thus determine

$$
\alpha_{0}=\left[\frac{3}{2} N \sum_{m=1}^{\infty} m z_{\Phi}\left(e^{-m \beta}\right)\right]^{-1 / 2} .
$$

For one-harmonic case, this gives $\alpha_{0}=\left[\frac{3}{2} N z_{\Phi}\left(e^{-\beta}\right)\right]^{-1 / 2}$ or $u=1 /\left(6 N z_{\Phi}\right)+\ldots$, in agreement with (4.37).

\section{C.4 Eigenvalue density for (anti) symmetric 3-plet representation}

We can repeat the analysis of section 4 for the (anti) symmetric 3-plet representation, see (2.11). Here the action is (4.8) with

$$
\mathcal{V}^{ \pm}(\boldsymbol{\alpha})=\frac{1}{3} \sum_{i j k} \cos \left(\alpha_{i}+\alpha_{j}+\alpha_{k}\right) \pm \sum_{i j} \cos \left(\alpha_{i}+2 \alpha_{j}\right)+\frac{2}{3} \sum_{i} \cos \left(3 \alpha_{i}\right) .
$$

The stationary-point equation for the eigenvalues $\alpha_{i}$ is

$$
\begin{aligned}
\sum_{j \neq i} \cot \frac{\alpha_{i}-\alpha_{j}}{2}-\sum_{m=1}^{\infty} z_{\Phi}\left(e^{-m \beta}\right)[ & \sum_{j k} \sin \left(m\left(\alpha_{i}+\alpha_{j}+\alpha_{k}\right)\right) \pm \sum_{j} \sin \left(m\left(\alpha_{i}+2 \alpha_{j}\right)\right) \\
& \left. \pm 2 \sum_{j} \sin \left(m\left(2 \alpha_{i}+\alpha_{j}\right)\right)+2 \sin \left(3 m \alpha_{i}\right)\right]=0 .
\end{aligned}
$$


Written in terms of the density of eigenvalues (4.13), this becomes

$$
\begin{aligned}
& \int d \alpha^{\prime} \rho\left(\alpha^{\prime}\right) \cot \frac{\alpha-\alpha^{\prime}}{2}=\sum_{m=1}^{\infty} z_{\Phi}\left(e^{-m \beta}\right)\left[N \int d \alpha^{\prime} \int d \alpha^{\prime \prime} \rho\left(\alpha^{\prime}\right) \rho\left(\alpha^{\prime \prime}\right) \sin \left(m\left(\alpha+\alpha^{\prime}+\alpha^{\prime \prime}\right)\right)\right. \\
& \left.\left. \pm \int d \alpha^{\prime} \rho\left(\alpha^{\prime}\right) \sin \left(m\left(\alpha+2 \alpha^{\prime}\right)\right) \pm 2 \int d \alpha^{\prime} \rho\left(\alpha^{\prime}\right) \sin \left(m\left(2 \alpha+\alpha^{\prime}\right)\right)+\frac{2}{N} \sin (3 m \alpha)\right] . \quad \text { (C. } 21\right)
\end{aligned}
$$

Comparing to (4.28) found in the general 3-plet case we observe that the additional terms appearing in the (anti) symmetric case are suppressed at large $N$ by powers of $1 / N$.

In more detail, in the simple one-harmonic case, we can write (C.21) in the form of (4.29), (4.30)

$$
\int d \alpha^{\prime} \rho\left(\alpha^{\prime}\right) \cot \frac{\alpha-\alpha^{\prime}}{2}=2 \sum_{m=1}^{3} C_{m} \sin (m \alpha)
$$

where

$$
C_{1}=\frac{1}{2}\left(N \rho_{1}^{2} \pm \rho_{2}\right) z_{\Phi}, \quad C_{2}= \pm z_{\Phi} \rho_{1}, \quad C_{3}=\frac{z_{\Phi}}{N},
$$

and then the solution for the density is similar to (4.31), (4.32). Assuming that for $N \rightarrow \infty$ with $N z_{\Phi}$ fixed we have $\rho_{1}, \rho_{2}$ finite, it is clear that the effects of (anti) symmetrization are subleading.

To check these assumptions, let us consider explicitly the symmetric representation case, i.e. the plus sign in (C.21), (C.23). Introducing $u=\sin ^{2} \frac{\alpha_{0}}{2}$, the three self-consistency conditions obtained by plugging $\rho$ into the definition of $\rho_{1}$ and $\rho_{2}$ and also imposing $\int d \alpha \rho(\alpha)=1$ are

$$
\begin{aligned}
1= & \frac{2}{N} u\left(10 u^{2}-12 u+3\right) z_{\Phi}+N \rho_{1}^{2} u z_{\Phi}-u z_{\Phi}\left(-4 \rho_{1}-\rho_{2}+6 \rho_{1} u\right), \\
\rho_{1}= & -\frac{1}{2} N \rho_{1}^{2}(u-2) u z_{\Phi}-\frac{3}{N} u(5 u-2)(u-1)^{2} z_{\Phi} \\
& +\frac{1}{2} u z_{\Phi}\left(8 \rho_{1}+2 \rho_{2}+8 \rho_{1} u^{2}-16 \rho_{1} u-\rho_{2} u\right), \\
\rho_{2}= & \frac{6}{N} u\left(6 u^{2}-4 u+1\right)(u-1)^{2} z_{\Phi}+N \rho_{1}^{2} u(u-1)^{2} z_{\Phi} \\
& -u z_{\Phi}\left(-4 \rho_{1}-\rho_{2}+9 \rho_{1} u^{3}-20 \rho_{1} u^{2}-\rho_{2} u^{2}+14 \rho_{1} u+2 \rho_{2} u\right) .
\end{aligned}
$$

One may study the properties of the solution $\left(u, \rho_{1}, \rho_{2}\right)$ of the algebraic system (C.24) for fixed $z_{\Phi}$ and increasing $N$. A numerical analysis shows that for any $z_{\Phi}$ we find exactly one acceptable solution as soon as $N$ is sufficiently large. This solution may be expanded in powers of $1 / N$ and reads

$$
\begin{aligned}
u & =\frac{1}{N z_{\Phi}}+\frac{1}{\left(N z_{\Phi}\right)^{2}}-\frac{5}{N} \frac{1}{N z_{\Phi}}+\ldots \\
\rho_{1} & =1-\frac{1}{2 N z_{\Phi}}-\frac{1}{2\left(N z_{\Phi}\right)^{2}}+\frac{5}{2 N} \frac{1}{N z_{\Phi}}+\ldots \\
\rho_{2} & =1-\frac{1}{2 N z_{\Phi}}-\frac{1}{\left(N z_{\Phi}\right)^{2}}+\frac{10}{N} \frac{1}{N z_{\Phi}}+\ldots
\end{aligned}
$$




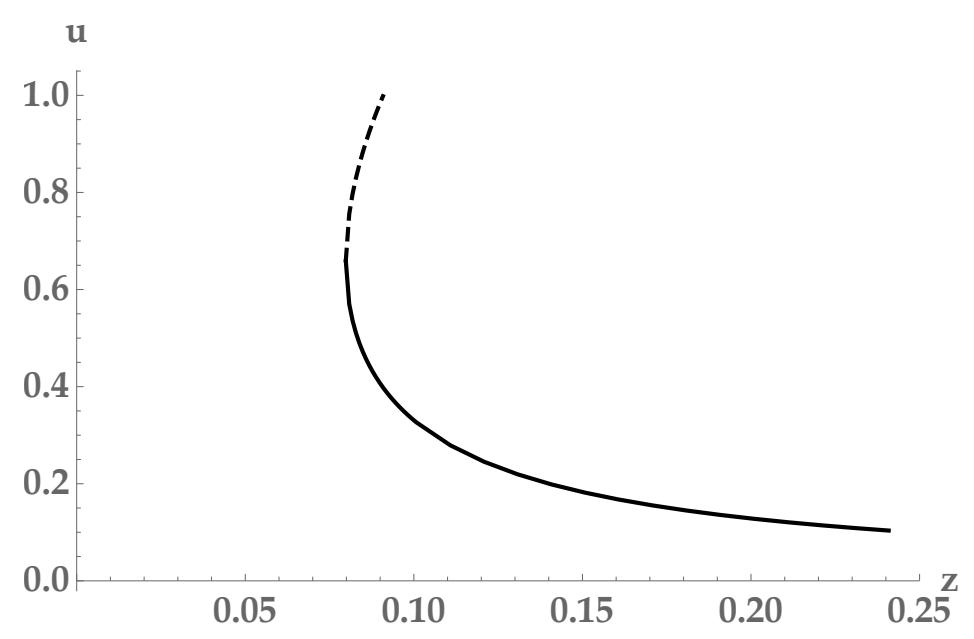

Figure 5. Solution of the system (C.24) for $u$ as a function of variable $z \equiv z_{\Phi}$ for $N=40$.

Thus the large $N$ behaviour at fixed $N z_{\Phi}$ is similar to the asymmetric 3-plet case, with $\alpha_{0} \sim\left(N z_{\Phi}\right)^{-1 / 2}$. If we fix $N$ and vary $z_{\Phi}$, the solution may develop branches and may exist only in certain ranges. One example is in figure 5 where we show the solution for $u$ as a function of $z_{\Phi}$ for $N=40$. There is a minimal value of $z_{\Phi}$ and also a narrow region where both branches are present. Completely similar features are observed in the case of the antisymmetric 3-plet representation.

\section{D $\quad N=\infty$ limit of low temperature expansion of $O(N)$ partition func- tion}

If the symmetry group is $O(N)$, we may again start with the general expression for the partition function in (2.7). Renaming matrix $U$ as $M \in O(N)$, the characters of the relevant representations $R$ are

\begin{tabular}{|cc|}
\hline$R$ & $\chi_{R}$ \\
\hline vector $: N$ & $\operatorname{tr}(M)$ \\
adjoint $:(N \otimes N)_{A}$ & $\frac{1}{2} \operatorname{tr}(M)^{2}-\frac{1}{2} \operatorname{tr}\left(M^{2}\right)$ \\
3-plet $: N^{\otimes 3}$ & $\operatorname{tr}(M)^{3}$ \\
\hline
\end{tabular}

Expanding (2.7) in powers of the matrix $M$, we are led to the problem of computing the $O(N)$ group integrals parametrized by the integers $\boldsymbol{a}=\left\{a_{\ell}\right\}$

$$
I(\boldsymbol{a})=\int d M \prod_{\ell \geq 1}\left(\operatorname{tr} M^{\ell}\right)^{a_{\ell}} .
$$

As in the $\mathrm{U}(N)$ case in (3.6), if $N$ is sufficiently large, $I(\boldsymbol{a})$ does not depend on $N$ and factorizes. The precise condition is $N \geq 2 \kappa(\boldsymbol{a})$ where $\kappa(\boldsymbol{a})$ was defined in (3.7). In this 
case one can represent the integral in the form

$$
I(\boldsymbol{a})=\int \prod_{\ell} \frac{d \xi_{\ell}}{\sqrt{2 \pi}} e^{-\frac{1}{2} \xi_{\ell}^{2}} \prod_{\ell \geq 1}\left(\sqrt{\ell} \xi_{\ell}+\eta_{\ell}\right)^{a_{\ell}}, \quad \eta_{l}=\frac{1+(-1)^{\ell}}{2},
$$

where $\xi_{\ell}$ are independent normal variables with Gaussian distribution [51]. As a result,

$$
I(\boldsymbol{a})=\prod_{\ell \geq 1} \begin{cases}\frac{1+(-1)^{a_{\ell}}}{2}(2 \ell)^{a_{\ell} / 2} \frac{1}{\sqrt{\pi}} \Gamma\left(\frac{a_{\ell}+1}{2}\right), & \ell \text { odd }, \\
\sum_{n=0}^{a_{\ell}}\left(\begin{array}{c}
a_{\ell} \\
n
\end{array}\right) \frac{1+(-1)^{n}}{2}(2 \ell)^{n / 2} \frac{1}{\sqrt{\pi}} \Gamma\left(\frac{n+1}{2}\right), & \ell \text { even. }\end{cases}
$$

Using this result, we may determine the low temperature expansion of the partition function for a $4 \mathrm{~d}$ scalar field transforming in various representations like in (D.1). In the vector representation case we get (cf. (3.10))

$$
Z_{\mathrm{S}, 4}^{\mathrm{vector}}=1+x^{2}+4 x^{3}+20 x^{4}+56 x^{5}+164 x^{6}+412 x^{7}+1116 x^{8}+\ldots
$$

This agrees with the result of [12] for the large $N$ partition function in the $O(N)$ case which is given by (3.1) with the following "single-trace" partition function (cf. (3.3) $)^{24}$

$$
\begin{aligned}
Z_{\mathrm{s.t.}}^{\text {vector }} & =\frac{1}{2}\left[z_{\Phi}(x)\right]^{2}+\frac{1}{2} z_{\Phi}\left(x^{2}\right) \\
& =x^{2}+4 x^{3}+19 x^{4}+52 x^{5}+134 x^{6}+280 x^{7}+554 x^{8}+984 x^{9}+\ldots
\end{aligned}
$$

In the adjoint scalar case, we find (cf. (3.11))

$$
\begin{aligned}
Z_{\mathrm{S}, 4}^{\text {adjoint }}= & 1+x^{2}+4 x^{3}+21 x^{4}+66 x^{5}+235 x^{6}+724 x^{7}+2423 x^{8}+7873 x^{9} \\
& +26463 x^{10}+88252 x^{11}+297918 x^{12}+1003530 x^{13}+\ldots,
\end{aligned}
$$

with the corresponding single-trace partition function in (3.1) being

$$
\begin{aligned}
Z_{\mathrm{s.t.}}^{\text {adjoint }}= & x^{2}+4 x^{3}+20 x^{4}+62 x^{5}+204 x^{6}+578 x^{7}+1730 x^{8} \\
& +5073 x^{9}+15495 x^{10}+47791 x^{11}+\ldots
\end{aligned}
$$

$Z_{\mathrm{s.t}}^{\text {adjoint }}$ counts the operators which are single traces of products of scalars fields which are antisymmetric $O(N)$ matrices. Here we have the identity

$$
\operatorname{tr}\left(\Phi_{1} \Phi_{2} \ldots \Phi_{n}\right)=(-1)^{n} \operatorname{tr}\left(\Phi_{n} \Phi_{n-1} \ldots \Phi_{1}\right)
$$

where $\Phi_{n}$ is the scalar $\varphi_{i j}=-\varphi_{j i}$ or any derivative of it. It seems non-trivial to apply Polya counting in the case of an additional constraint (D.9), and we did not find a simple closed formula for $Z_{\text {s.t. }}$ like (3.4). To see the non-trivial effect of the constraint (D.9), let

\footnotetext{
${ }^{24}$ Here the scalar is real so the $x^{2}$ term corresponds to the operator $\varphi_{i} \varphi_{i}$. The coefficient 4 of $x^{3}$ term comes from $\varphi_{i} \partial_{\mu} \varphi_{i}$. The $19 x^{4}$ term comes from 9 operators $\varphi_{i} \partial_{\mu} \partial_{\nu} \varphi_{i}$ and $\frac{1}{2}(4 \times 5)=10$ operators $\partial_{\mu} \varphi_{i} \partial_{\nu} \varphi_{i}$, etc.
} 
us explicitly list the single-trace operators up to dimension 5:

\begin{tabular}{|ccc|}
\hline $\operatorname{dim}$ & operator & multiplicity \\
\hline 2 & $\operatorname{tr}(\varphi \varphi)$ & 1 \\
\hline 3 & $\operatorname{tr}\left(\varphi \partial_{\mu} \varphi\right)$ & 4 \\
\hline 4 & $\operatorname{tr}\left(\varphi \partial_{\mu} \partial_{\nu} \varphi\right)$ & 9 \\
& $\operatorname{tr}\left(\partial_{\mu} \varphi \partial_{\nu} \varphi\right)$ & 10 \\
& $\operatorname{tr}(\varphi \varphi \varphi \varphi)$ & 1 \\
\hline 5 & $\operatorname{tr}\left(\varphi \partial_{\mu} \partial_{\nu} \partial_{\rho} \varphi\right)$ & $20-4=16$ \\
& $\operatorname{tr}\left(\partial_{\mu} \varphi \partial_{\nu} \partial_{\rho} \varphi\right)$ & $4 \times 9=36$ \\
& $\operatorname{tr}\left(\varphi \partial_{\mu} \varphi \partial_{\nu} \varphi\right)$ & $6(\mu \neq \nu)$ \\
& $\operatorname{tr}\left(\varphi \varphi \varphi \partial_{\mu} \varphi\right)$ & 4 \\
\hline
\end{tabular}

Note that $\operatorname{tr}\left(\varphi \varphi \partial_{\mu} \partial_{\nu} \varphi\right)=0$ in view of (D.9). Also, $\operatorname{tr}\left(\varphi \partial_{\mu} \varphi \partial_{\nu} \varphi\right)$ with $\mu=\nu$ is again zero in view of (D.9). The resulting multiplicities 1, 4, 20, 62 are in agreement with (D.8).

The case the symmetric representation $(N \otimes N)_{S}$ appears to be simpler. Here the analog of (D.9) reads

$$
\operatorname{tr}\left(\Phi_{1} \Phi_{2} \ldots \Phi_{n}\right)=\operatorname{tr}\left(\Phi_{n} \Phi_{n-1} \ldots \Phi_{1}\right)
$$

and it adds an extra symmetry to the standard cyclic invariance of the trace. Then the total and single-trace partition functions are found to be

$$
\begin{aligned}
Z_{\mathrm{S}, 4}^{2 \text {-plet }^{+}}= & 1+x+6 x^{2}+20 x^{3}+75 x^{4}+246 x^{5}+862 x^{6}+2852 x^{7} \\
& +9643 x^{8}+32040 x^{9}+107141 x^{10}+356651 x^{11}+1191345 x^{12}+\ldots, \\
Z_{\text {s.t. }}^{\text {2-plet }}= & x+5 x^{2}+14 x^{3}+40 x^{4}+101 x^{5}+276 x^{6}+715 x^{7} \\
& +1982 x^{8}+5553 x^{9}+16379 x^{10}+49476 x^{11}+154346 x^{12}+\ldots
\end{aligned}
$$

One can find a closed form of (D.13) using the Polya enumeration theorem and taking into account that the symmetry group is the cyclic group with an additional inversion (D.11). A careful examination of the cycle structure of the associated permutations gives

$$
\begin{aligned}
Z_{\text {s.t. }}^{2 \text {-plet }}= & -\frac{1}{2} \sum_{m=1}^{\infty} \frac{\varphi(m)}{m} \log \left(1-z_{\Phi}\left(x^{m}\right)\right) \\
& +\frac{1}{2} \sum_{m=1}^{\infty} \sum_{\ell=1}^{k} \frac{1}{m} \begin{cases}\left\{\begin{array}{l}
{\left[z_{\Phi}\left(x^{2}\right)\right]^{\frac{m}{2}}} \\
{\left[z_{\Phi}\left(x^{2}\right)\right]^{\frac{m}{2}}-1}
\end{array} z_{\Phi}(x)\right]^{2} & \ell \text { odd } \\
{\left[z_{\Phi}\left(x^{2}\right)\right]^{\frac{m-1}{2}} z_{\Phi}(x)} & m \text { even }\end{cases}
\end{aligned}
$$

where $\varphi(m)$ is the same as in (3.4) and the additional $1 / 2$ factors are due to the fact that the symmetry group for a trace with $m$ objects is $2 m$ (from $m$ shifts and $m$ reflected shifts). ${ }^{25}$

\footnotetext{
${ }^{25}$ The presence of extra terms in the second line of (D.14) is due to the fact that a reflected shift by $\ell$ places of a string of $m$ objects splits into: (i) $\frac{m}{2}$ 2-cycles if $\ell, q$ are even; (ii) $\frac{m-2}{2} 2$-cycles and 2 -cycles if $\ell$ is odd and $m$ is even; (iii) $\frac{m-1}{2}$ 2-cycles and one 1-cycle if $m$ is odd.
} 
Using (D.14), we have computed the series expansion (D.13) up to the very high order $\mathcal{O}\left(x^{100}\right)$ and a numerical analysis revealed that the series is convergent for $x<x_{c}$ with $z_{\Phi}\left(x_{c}\right)=1$ (for the $4 \mathrm{~d}$ scalar this critical value is $x_{c} \simeq 0.285$ ). The same behaviour was found in the $\mathrm{U}(N)$ case so the conclusion is that the additional terms in the second line of (D.14) do not worsen the convergence.

In the 3-plet case representation case we obtain (cf. (3.10))

$$
\begin{aligned}
Z_{\mathrm{S}, 4}^{3 \text {-plet }}= & 1+11 x^{2}+60 x^{3}+773 x^{4}+7920 x^{5}+110781 x^{6}+1509060 x^{7} \\
& +23807838 x^{8}+379566780 x^{9}+6645202174 x^{10}+118587559020 x^{11} \\
& +2264713625957 x^{12}+44204970285420 x^{13}+\ldots,
\end{aligned}
$$

with the corresponding "single-trace" partition function being

$$
\begin{aligned}
Z_{\mathrm{s.t.}}^{3-\text { plet }}= & 11 x^{2}+60 x^{3}+707 x^{4}+7260 x^{5}+100888 x^{6}+1382820 x^{7}+21944399 x^{8} \\
& +352168900 x^{9}+6207336278 x^{10}+111438968700 x^{11}+\ldots
\end{aligned}
$$

To reproduce the $x^{2}$ term here by counting dimension 2 operators we need to classify various bilinear contractions: (i) there are $\frac{1}{2}(3 \times 4)=6$ contractions containing traces $\varphi_{i i j} \varphi_{i i j}, \varphi_{i i j} \varphi_{i j i}, \ldots$, where we need to account that position of the index contracted between the two fields matters and that there is a symmetry between the two fields in the real scalar case; (ii) there are also 3 ! irreducible contractions $\varphi_{i j k} \varphi_{i j k}, \varphi_{i j k} \varphi_{i k j}, \ldots$, but one needs to take into account the symmetry relation $\varphi_{i j k} \varphi_{j k i} \equiv \varphi_{i j k} \varphi_{k i j}$, so we are left with $6-1=5$ independent choices. The total $6+5=11$ matches the coefficient of the $x^{2}$ term in (D.15), (D.16).

There are fewer operators in the case of totally symmetric or antisymmetric 3-plet representations, i.e. the coefficients in the small $x$ expansion of $Z$ should be much smaller. Indeed, we find directly from (2.11) (cf. (3.19), (3.20))

$$
\begin{aligned}
Z_{\mathrm{S}, 4}^{3 \text {-plet }^{+}=} & 1+2 x^{2}+8 x^{3}+46 x^{4}+156 x^{5}+668 x^{6}+2684 x^{7}+12044 x^{8} \\
& +53556 x^{9}+249495 x^{10}+1182524 x^{11}+5780012 x^{12}+\ldots, \\
Z_{\text {s.t. }}^{3 \text {-plet }^{+}=} & 2 x^{2}+8 x^{3}+43 x^{4}+140 x^{5}+542 x^{6}+2036 x^{7}+8688 x^{8} \\
& +37868 x^{9}+175063 x^{10}+832556 x^{11}+4102153 x^{12}+\ldots, \\
Z_{\mathrm{S}, 4}^{3-\text { plet }^{-}=} & 1+x^{2}+4 x^{3}+22 x^{4}+64 x^{5}+228 x^{6}+716 x^{7}+2701 x^{8} \\
& +10104 x^{9}+41897 x^{10}+179420 x^{11}+820244 x^{12}+\ldots, \\
Z_{\text {s.t. }}^{3 \text {-plet }^{-}=} & x^{2}+4 x^{3}+21 x^{4}+60 x^{5}+196 x^{6}+568 x^{7}+2002 x^{8} \\
& +7324 x^{9}+30768 x^{10}+136096 x^{11}+644817 x^{12}+\ldots
\end{aligned}
$$


For example, the first few single-trace states in the antisymmetric case are

\begin{tabular}{|ccc|}
\hline $\operatorname{dim}$ & operator & multiplicity \\
\hline 2 & $\varphi_{i j k} \varphi_{i j k}$ & 1 \\
\hline 3 & $\varphi_{i j k} \partial_{\mu} \varphi_{i j k}$ & 4 \\
\hline 4 & $\varphi_{i j k} \partial_{\mu} \partial_{\nu} \varphi_{i j k}$ & 9 \\
& $\partial_{\mu} \varphi_{i j k} \partial_{\nu} \varphi_{i j k}$ & 10 \\
& $\varphi_{i j k} \varphi_{i j l} \varphi_{p q k} \varphi_{p q l}$ & 1 \\
& $\varphi_{i j k} \varphi_{i p q} \varphi_{j p l} \varphi_{k q l}$ & 1 \\
\hline
\end{tabular}

As a final remark, we note that using the discussion in [12] providing the suitable modification of the measure term in (4.6) for the $O(N)$ case, it is possible also to study the large $N$ thermodynamics and the structure of phase transitions in this case, but there should be no qualitative changes compared to $\mathrm{U}(N)$ case analyzed in section 4 .

\section{E General expression for single-trace partition function}

Given the $N=\infty$ partition function $Z(x)$ one can invert the relation (3.1) and find the single-trace partition function $Z_{\text {s.t. }}(x)$ that counts only irreducible ("single-trace") contractions among all singlet operators. Starting with the relation

$$
L(x)=\sum_{m=1}^{\infty} \frac{1}{m} Z_{\text {s.t. }}\left(x^{m}\right), \quad L(x) \equiv \log Z(x),
$$

we find

$$
Z_{\text {s.t. }}(x)=L(x)+\sum_{m \in \Omega}(-1)^{\nu_{m}} \frac{1}{m} L\left(x^{m}\right) .
$$

Here $\Omega=\{2,3,5,6,7,10,11,13, \ldots\}$ is the set of so-called square-free integers, such that their prime number factorization is of the form $m=\prod_{i=1}^{\nu_{m}} p_{i}$, i.e. is the product of prime factors each appearing in the first power only. The sign factor $(-1)^{\nu_{m}}$ is known in this context as the Liouville function. The proof of (E.2) is by substituting (E.2) into (E.1):

$$
\begin{aligned}
& \sum_{m=1}^{\infty} \frac{1}{m} Z_{\text {s.t. }}\left(x^{m}\right)=\sum_{m=1}^{\infty} \frac{1}{m} L\left(x^{m}\right)+\sum_{n=1}^{\infty} \sum_{q \in \Omega}(-1)^{\nu_{q}} \frac{1}{n q} L\left(x^{n q}\right) \\
& =\sum_{m=1}^{\infty} \frac{1}{m} L\left(x^{m}\right)\left[1+\sum_{\substack{q \in \Omega \\
q \mid m}}(-1)^{\nu_{q}}\right]=L(x) .
\end{aligned}
$$

In the last equality, we used that

$$
\sum_{\substack{q \in \Omega \\ q \mid m}}(-1)^{\nu_{q}}= \begin{cases}1, & m=1 \\ 0, & m>1 .\end{cases}
$$


To prove (E.4), it is enough to observe that if $m>1$ is factorizable into $M$ powers of distinct primes, $m=p_{1}^{a_{1}} \cdots p_{M}^{a_{M}}$, then

$$
1+\sum_{\substack{q \in \Omega \\ q \mid m}}(-1)^{\nu_{q}}=1-M+\frac{M(M-1)}{2}-\frac{M(M-1)(M-2)}{3 !}+\ldots=(1-1)^{M}=0
$$

where we used that the square-free integers $q$ dividing $m$ are of the form $p_{i}, p_{i} p_{j}(i \neq j)$, etc.

Let us note that if $L(x)$ in (E.1) has the form

$$
L(x)=\sum_{m=1}^{\infty} f\left(x^{m}\right)
$$

where $f(x)$ is independent of $m$, then one can show that (E.2) gives

$$
Z_{\text {s.t. }}(x)=\sum_{m=1}^{\infty} \frac{\varphi(m)}{m} f\left(x^{m}\right),
$$

where $\varphi$ is the Euler's totient function as in (3.4). One example is the adjoint representation case in (3.5). Another is the 2-plet representation with $Z$ given in (A.3) in which case (cf. $(3.4))$

$$
Z_{\text {s.t. }}^{2 \text {-plet }}(x)=-\frac{1}{2} \sum_{m=1}^{\infty} \frac{\varphi(m)}{m} \log \left(1-4\left[z_{\Phi}\left(x^{m}\right)\right]^{2}\right) \text {. }
$$

\section{F Singlet partition function of $[\mathrm{U}(N)]^{p}$ invariant $p$-tensor theory}

Given a free $p$-tensor $\Phi=\left(\varphi_{i_{1} \ldots i_{p}}\right)$ with each internal index running from from 1 to $N$ one may have several options of how to define the corresponding CFT and thus the associated singlet partition function $Z(x)$ (i.e. which singlet operators given by contractions of fields to include). In the main part of this paper we treated all $p$ indices of $\left(\varphi_{i_{1} \ldots i_{p}}\right)$ as equivalent and thus all of their contractions were allowed. The corresponding singlet partition function on $S_{\beta}^{1} \times S^{d-1}$ was then found by gauging the global $\mathrm{U}(N)$ or $O(N)$ symmetry.

If instead all $p$ indices are assumed to be distinguishable as, e.g., in the interacting tensor models considered in [33, 37], then the singlet constraint may be implemented by gauging the full $[\mathrm{U}(N)]^{p}$ symmetry group [33]. As we shall demonstrate below, in this case the low temperature expansion of $Z(x)$ will again diverge in the $N=\infty$ limit starting with the $p=3$ case, i.e. the critical temperature will vanish with $N \rightarrow \infty$ if $p \geq 3 .^{26}$

The computation of the singlet partition function in the $[\mathrm{U}(N)]^{p}$ invariant theory (that we will call " $p$-tensor" theory for short) turns out to be very similar to the case of the $\mathrm{U}(N)$ invariant $p$-plet theory considered in sections 3 and 4 above. To compute $Z$ we may start with the path integral for $\varphi_{i_{1} \ldots i_{p}}$ with covariant derivative containing $p$ independent flat gauge fields $A_{\mu}^{(r)}(r=1, \ldots, p)$ and average over their $S^{1}$ holonomies, i.e. constant $N \times N$ hermitian $A_{0}^{(r)}$ matrices with the eigenvalues $\alpha_{i r}$, or, equivalently,

\footnotetext{
${ }^{26}$ We are thank I. Klebanov and G. Tarnopolsky for the suggestion to investigate this case.
} 
over $\mathrm{U}(N)$ matrices $U_{r}$ with eigenvalues $e^{i \alpha_{i r}}(i=1, \ldots, N ; r=1, \ldots, p)$. As $\varphi_{i_{1} \ldots i_{p}}$ transforms in the direct product of fundamental representations of $p$ copies of $\mathrm{U}(N)$ group, the potentials $A_{0}^{(r)}$ or the eigenvalues $\alpha_{i r}$ simply sum up, i.e. the resulting partition function will be a straightforward generalization of (2.7) for the fundamental representation of a single $\mathrm{U}(N)$ with the character of the real representation $R$ in (2.7) now being

$$
\chi\left(U_{1}, \ldots, U_{p}\right)=\prod_{r=1}^{p} \operatorname{tr} U_{r}+\prod_{r=1}^{p} \operatorname{tr} U_{r}^{-1} .
$$

Considering the case of low temperature expansion of $Z$ in the $N=\infty$ limit one finds, doing $p$ independent $d U_{1} \cdots d U_{p}$ integrations in the same way as in $\mathrm{U}(N)$-plet case in $(3.24),(3.25)$,

$$
Z^{p \text {-tensor }}=\prod_{m=1}^{\infty} \sum_{k=0}^{\infty} \frac{1}{(2 k) !}\left(\frac{z_{\Phi}\left(x^{m}\right)}{m}\right)^{2 k}\left(\begin{array}{c}
2 k \\
k
\end{array}\right) m^{p k}(k !)^{p}=\prod_{m=1}^{\infty} G_{p}\left(m^{p-2}\left[z_{\Phi}\left(x^{m}\right)\right]^{2}\right),
$$

where here the role of $F_{p}$ in (3.25), (3.26) is played by the power series

$$
G_{p}(y)=\sum_{k=0}^{\infty} g_{k} y^{k}, \quad g_{k}=(k !)^{p-2}, \quad p=1,2,3, \ldots
$$

As (F.2) involves the square of $z_{\Phi}$ and thus is not sensitive to sign factor in (2.8) it looks the same for both pure boson or pure fermion cases.

The $p=1$ is of course the standard vector or 1-plet case when $G_{1}(y)=F_{1}(y)=e^{y}$ as in (3.27), (3.28). In the 2 -tensor case we get $G_{2}(y)=1 /(1-y)$ (cf. (3.27)) and thus

$$
Z^{2 \text {-tensor }}=\prod_{m=1}^{\infty}\left(1-\left[z_{\Phi}\left(x^{m}\right)\right]^{2}\right)^{-1}
$$

This is similar to the adjoint $\mathrm{U}(N)$ case (3.5) (with $\left.z_{\Phi} \rightarrow z_{\Phi}^{2}\right)^{27}$ and also to the 2-plet case (3.29), (A.3).

As in the $p$-plet case in (3.26), the $p=3$ is the critical value: since $g_{k+1} / g_{k}=(k+1)^{p-2}$ the series $G_{p}(y)$ in (F.3) does not converge for $p \geq 3$. The function

$$
G_{3}(y)=\sum_{k=0}^{\infty} k ! y^{k}
$$

that has zero radius of convergence can be Borel-resummed for $y<0$ giving (cf. (3.31))

$$
\widetilde{G}_{3}^{B}(y)=-y^{-1} e^{-y^{-1}} \Gamma\left(0,-y^{-1}\right),
$$

where $\Gamma(s, y)=\int_{y}^{\infty} d t e^{-t} t^{s-1}$ is the incomplete $\Gamma$ function. Thus $G_{3}(y)$ is an asymptotic expansion of $\widetilde{G}_{3}^{B}(y)$ for $y<0$. $\widetilde{G}_{B}(y>0)$ has an imaginary part $\frac{\pi}{y} e^{-1 / y}$ that vanishes exponentially fast for $y \rightarrow 0^{+}$.

\footnotetext{
${ }^{27}$ This relation can be understood in terms of counting operators as follows: each singlet can be considered as built out of elementary fields $P_{i j}=\bar{\varphi}_{i k} \varphi_{j k}$ (with possible derivatives) with these $P$-fields contracted in a matrix-like style. Alternatively, one may build all singlets using the basis of $P_{i j}^{\prime}=\bar{\varphi}_{k i} \varphi_{k j}$.
} 
For example, in the case of a 3 -tensor field $\Phi$ being a 4 d scalar we find from (F.2), (F.3)

$$
\begin{aligned}
Z_{\mathrm{S}, 4}^{3 \text {-tensor }}= & 1+x^{2}+8 x^{3}+38 x^{4}+136 x^{5}+550 x^{6} \\
& +2224 x^{7}+9727 x^{8}+42592 x^{9}+191836 x^{10}+\ldots
\end{aligned}
$$

Comparing this to the 3-plet case in (3.12) we see much smaller coefficients, i.e. the number of singlet operators is reduced at each order in dimension. ${ }^{28}$

The single-trace partition function in (3.1), (E.2) corresponding to (F.7) is

$$
Z_{\text {s.t. }}^{3 \text {-tensor }}=x^{2}+8 x^{3}+37 x^{4}+128 x^{5}+476 x^{6}+1792 x^{7}+7450 x^{8}+31704 x^{9}+\ldots,
$$

and the lowest coefficients here are reproduced by the operator counting as follows:

\begin{tabular}{|ccc|}
\hline $\operatorname{dim}$ & operator & multiplicity \\
\hline 2 & $\bar{\varphi}_{i j k} \varphi_{i j k}$ & 1 \\
\hline 3 & $\bar{\varphi}_{i j k} \partial_{\mu} \varphi_{i j k}$ and c.c. & $2 \times 4=8$ \\
\hline 4 & $\bar{\varphi}_{i j k} \partial_{\mu} \partial_{\nu} \varphi_{i j k}$ and c.c. & $2 \times 9=18$ \\
& $\partial_{\mu} \bar{\varphi}_{i j k} \partial_{\nu} \varphi_{i j k}$ & $4 \times 4=16$ \\
& $\bar{\varphi}_{i j k} \varphi_{i r s} \bar{\varphi}_{l r s} \varphi_{l j k}$ & 3 \\
\hline
\end{tabular}

where the multiplicity 3 in last row corresponds to the position of the index contracted between $\bar{\varphi}$ and $\varphi$.

As in the 3-plet case discussed in section 4 , the zero radius of convergence of the low temperature series for the $[\mathrm{U}(N)]^{3}$ singlet partition function $Z^{3 \text {-tensor }}$ at $N=\infty$ should be related to the vanishing of the corresponding critical temperature in the limit $N \rightarrow \infty$. This can be seen explicitly by repeating the analysis in section 4 in the 3 -tensor case. Here we will have 3 sets of eigenvalues $\alpha_{i r}(r=1,2,3)$ and thus 3 densities $\rho_{r}(\alpha)$ with the analog of the action (4.14), (4.15), (4.19) being

$$
\begin{aligned}
S\left(\rho_{r}, x\right)= & N^{2} \sum_{r=1}^{3} \int d \alpha d \alpha^{\prime} K\left(\alpha-\alpha^{\prime}\right) \rho_{r}(\alpha) \rho_{r}\left(\alpha^{\prime}\right) \\
& -2 N^{3} \int d \alpha d \alpha^{\prime} d \alpha^{\prime \prime} \rho_{1}(\alpha) \rho_{2}\left(\alpha^{\prime}\right) \rho_{3}\left(\alpha^{\prime \prime}\right) \sum_{m=1}^{\infty} \frac{1}{m} z_{\Phi}\left(x^{m}\right) \cos \left[m\left(\alpha+\alpha^{\prime}+\alpha^{\prime \prime}\right)\right] .
\end{aligned}
$$

It is natural to look for a stationary point solution with the three equal densities $\rho_{1}=\rho_{2}=$ $\rho_{3} \equiv \rho(\alpha)$ for the three $\mathrm{U}(N)$ groups, thus getting the equation

$$
\int d \alpha^{\prime} \rho\left(\alpha^{\prime}\right) \cot \frac{\alpha-\alpha^{\prime}}{2}=2 N \sum_{m=1}^{\infty} z_{\Phi}\left(x^{m}\right) \int d \alpha^{\prime} d \alpha^{\prime \prime} \rho\left(\alpha^{\prime}\right) \rho\left(\alpha^{\prime \prime}\right) \sin \left[m\left(\alpha+\alpha^{\prime}+\alpha^{\prime \prime}\right)\right] .
$$

This equation is the same as in (4.28) up to a factor of 3 in the r.h.s. and thus its analysis is similar, implying that the critical temperature should again scale with $N$ as $T_{c} \sim(\log N)^{-1}$.

\footnotetext{
${ }^{28}$ Following the remark in footnote 14 , one can evaluate (F.2) with $z_{\Phi}(x) \rightarrow x$ corresponding to partition function of a constant scalar field in $4 \mathrm{~d}$. The resulting analog (or "truncation") of (F.7) will be a series in $x^{2}$ with the coefficients given by the known integer sequence A110143 http://oeis.org/A110143, see also eq. (19) of [52]. These coefficients have asymptotic factorial growth implying again zero radius of convergence.
} 
Open Access. This article is distributed under the terms of the Creative Commons Attribution License (CC-BY 4.0), which permits any use, distribution and reproduction in any medium, provided the original author(s) and source are credited.

\section{References}

[1] I.R. Klebanov and A.M. Polyakov, AdS dual of the critical $O(N)$ vector model, Phys. Lett. B 550 (2002) 213 [hep-th/0210114] [INSPIRE].

[2] S. Giombi, Higher spin-CFT duality, in Proceedings, Theoretical Advanced Study Institute in Elementary Particle Physics: New Frontiers in Fields and Strings (TASI 2015), Boulder CO U.S.A., 1-26 June 2015, pg. 137 [arXiv:1607.02967] [INSPIRE].

[3] S. Giombi, I.R. Klebanov and Z.M. Tan, The ABC of higher-spin AdS/CFT, arXiv:1608.07611 [INSPIRE].

[4] M. Beccaria and A.A. Tseytlin, Higher spins in $A d S_{5}$ at one loop: vacuum energy, boundary conformal anomalies and AdS/CFT, JHEP 11 (2014) 114 [arXiv:1410.3273] [INSPIRE].

[5] B. Sundborg, Stringy gravity, interacting tensionless strings and massless higher spins, Nucl. Phys. Proc. Suppl. 102 (2001) 113 [hep-th/0103247] [INSPIRE].

[6] P. Haggi-Mani and B. Sundborg, Free large- $N$ supersymmetric Yang-Mills theory as a string theory, JHEP 04 (2000) 031 [hep-th/0002189] [INSPIRE].

[7] J.-B. Bae, E. Joung and S. Lal, One-loop test of free $\mathrm{SU}(N)$ adjoint model holography, JHEP 04 (2016) 061 [arXiv: 1603.05387] [INSPIRE].

[8] J.-B. Bae, E. Joung and S. Lal, On the holography of free Yang-Mills, JHEP 10 (2016) 074 [arXiv: 1607.07651] [INSPIRE].

[9] J.-B. Bae, E. Joung and S. Lal, One-loop free energy of tensionless type IIB string in $A d S_{5} \times S^{5}, \operatorname{arXiv}: 1701.01507$ [INSPIRE].

[10] S.H. Shenker and X. Yin, Vector models in the singlet sector at finite temperature, arXiv:1109.3519 [INSPIRE].

[11] A. Jevicki, K. Jin and J. Yoon, $1 / N$ and loop corrections in higher spin $A d S_{4} / C F T_{3}$ duality, Phys. Rev. D 89 (2014) 085039 [arXiv:1401.3318] [InSPIRE].

[12] S. Giombi, I.R. Klebanov and A.A. Tseytlin, Partition functions and Casimir energies in higher spin $A d S_{d+1} / C F T_{d}$, Phys. Rev. D 90 (2014) 024048 [arXiv: 1402.5396] [INSPIRE].

[13] B. Sundborg, The Hagedorn transition, deconfinement and $N=4$ SYM theory, Nucl. Phys. B 573 (2000) 349 [hep-th/9908001] [INSPIRE].

[14] A.M. Polyakov, Gauge fields and space-time, Int. J. Mod. Phys. A 17S1 (2002) 119 [hep-th/0110196] [INSPIRE].

[15] O. Aharony, J. Marsano, S. Minwalla, K. Papadodimas and M. Van Raamsdonk, The Hagedorn-deconfinement phase transition in weakly coupled large- $N$ gauge theories, Adv. Theor. Math. Phys. 8 (2004) 603 [hep-th/0310285] [INSPIRE].

[16] B.S. Skagerstam, On the large- $N_{c}$ limit of the $\mathrm{SU}\left(N_{c}\right)$ color quark-gluon partition function, Z. Phys. C 24 (1984) 97 [inSPIRE].

[17] H.J. Schnitzer, Confinement/deconfinement transition of large- $N$ gauge theories with $N_{f}$ fundamentals: $N_{f} / N$ finite, Nucl. Phys. B 695 (2004) 267 [hep-th/0402219] [INSPIRE]. 
[18] A. Barabanschikov, L. Grant, L.L. Huang and S. Raju, The spectrum of Yang-Mills on a sphere, JHEP 01 (2006) 160 [hep-th/0501063] [INSPIRE].

[19] I. Amado, B. Sundborg, L. Thorlacius and N. Wintergerst, Probing emergent geometry through phase transitions in free vector and matrix models, JHEP 02 (2017) 005 [arXiv: 1612.03009] [INSPIRE].

[20] I.R. Klebanov and A.A. Tseytlin, Entropy of near extremal black p-branes, Nucl. Phys. B 475 (1996) 164 [hep-th/9604089] [INSPIRE].

[21] I.R. Klebanov and A.A. Tseytlin, Intersecting M-branes as four-dimensional black holes, Nucl. Phys. B 475 (1996) 179 [hep-th/9604166] [INSPIRE].

[22] S.S. Gubser, I.R. Klebanov and A.A. Tseytlin, String theory and classical absorption by three-branes, Nucl. Phys. B 499 (1997) 217 [hep-th/9703040] [INSPIRE].

[23] N. Seiberg, Notes on theories with 16 supercharges, Nucl. Phys. Proc. Suppl. 67 (1998) 158 [hep-th/9705117] [INSPIRE].

[24] J.A. Harvey, R. Minasian and G.W. Moore, Non-Abelian tensor multiplet anomalies, JHEP 09 (1998) 004 [hep-th/9808060] [INSPIRE].

[25] S.S. Gubser, I.R. Klebanov and A.A. Tseytlin, Coupling constant dependence in the thermodynamics of $N=4$ supersymmetric Yang-Mills theory, Nucl. Phys. B 534 (1998) 202 [hep-th/9805156] [INSPIRE].

[26] O. Aharony, S.S. Gubser, J.M. Maldacena, H. Ooguri and Y. Oz, Large-N field theories, string theory and gravity, Phys. Rept. 323 (2000) 183 [hep-th/9905111] [INSPIRE].

[27] F. Bastianelli, S. Frolov and A.A. Tseytlin, Three point correlators of stress tensors in maximally supersymmetric conformal theories in $D=3$ and $D=6$, Nucl. Phys. B 578 (2000) 139 [hep-th/9911135] [INSPIRE].

[28] F. Bastianelli, S. Frolov and A.A. Tseytlin, Conformal anomaly of $(2,0)$ tensor multiplet in six-dimensions and AdS/CFT correspondence, JHEP 02 (2000) 013 [hep-th/0001041] [INSPIRE].

[29] A.A. Tseytlin, $R^{4}$ terms in 11 dimensions and conformal anomaly of $(2,0)$ theory, Nucl. Phys. B 584 (2000) 233 [hep-th/0005072] [INSPIRE].

[30] A. Tseytlin, $(2,0)$ superconformal theory on multiple M5-branes, talk at the $4^{\text {th }}$ Asia-Pacific Center for theoretical physics Winter School Strings and D-branes, Seoul South Korea, (2000).

[31] M. Beccaria, G. Macorini and A.A. Tseytlin, Supergravity one-loop corrections on $A d S_{7}$ and $A d S_{3}$, higher spins and AdS/CFT, Nucl. Phys. B 892 (2015) 211 [arXiv:1412.0489] [INSPIRE].

[32] M. Beccaria and A.A. Tseytlin, Conformal anomaly c-coefficients of superconformal $6 d$ theories, JHEP 01 (2016) 001 [arXiv:1510.02685] [INSPIRE].

[33] I.R. Klebanov and G. Tarnopolsky, Uncolored random tensors, melon diagrams and the Sachdev-Ye-Kitaev models, Phys. Rev. D 95 (2017) 046004 [arXiv: 1611.08915] [inSPIRE].

[34] R. Gurau, The 1/N expansion of colored tensor models, Annales Henri Poincaré 12 (2011) 829 [arXiv: 1011.2726] [INSPIRE].

[35] R. Gurau and J.P. Ryan, Colored tensor models - a review, SIGMA 8 (2012) 020 [arXiv: 1109.4812] [INSPIRE]. 
[36] S. Carrozza and A. Tanasa, $O(N)$ random tensor models, Lett. Math. Phys. 106 (2016) 1531 [arXiv: 1512.06718] [INSPIRE].

[37] E. Witten, An SYK-like model without disorder, arXiv:1610.09758 [INSPIRE].

[38] H. Samtleben, E. Sezgin and R. Wimmer, $(1,0)$ superconformal models in six dimensions, JHEP 12 (2011) 062 [arXiv:1108.4060] [INSPIRE].

[39] H. Samtleben, E. Sezgin, R. Wimmer and L. Wulff, New superconformal models in six dimensions: gauge group and representation structure, PoS (CORFU2011) 071 [arXiv: 1204.0542] [INSPIRE].

[40] R. Güven, Black p-brane solutions of D = 11 supergravity theory, Phys. Lett. B 276 (1992) 49 [INSPIRE].

[41] G.W. Gibbons and P.K. Townsend, Vacuum interpolation in supergravity via super p-branes, Phys. Rev. Lett. 71 (1993) 3754 [hep-th/9307049] [INSPIRE].

[42] D.M. Kaplan and J. Michelson, Zero modes for the $D=11$ membrane and five-brane, Phys. Rev. D 53 (1996) 3474 [hep-th/9510053] [INSPIRE].

[43] X. Bekaert, M. Henneaux and A. Sevrin, Deformations of chiral two forms in six-dimensions, Phys. Lett. B 468 (1999) 228 [hep-th/9909094] [INSPIRE].

[44] X. Bekaert, Interactions of chiral two forms, hep-th/9911109 [INSPIRE].

[45] H.J. Schnitzer, Confinement/deconfinement transition of large- $N$ gauge theories in perturbation theory with $N_{f}$ fundamentals: $N_{f} / N$ finite, hep-th/0612099 [INSPIRE].

[46] M. Beccaria and A.A. Tseytlin, Vectorial $A d S_{5} / C F T_{4}$ duality for spin-one boundary theory, J. Phys. A 47 (2014) 492001 [arXiv:1410.4457] [inSPIRE].

[47] D. Kutasov and F. Larsen, Partition sums and entropy bounds in weakly coupled CFT, JHEP 01 (2001) 001 [hep-th/0009244] [INSPIRE].

[48] G.W. Gibbons, M.J. Perry and C.N. Pope, Partition functions, the Bekenstein bound and temperature inversion in anti-de Sitter space and its conformal boundary, Phys. Rev. D 74 (2006) 084009 [hep-th/0606186] [INSPIRE].

[49] A.B. Balantekin, Character expansion for $\mathrm{U}(N)$ groups and $\mathrm{U}(N / m)$ supergroups, J. Math. Phys. 25 (1984) 2028 [INSPIRE].

[50] Z. Puchala and J. Miszczak, Symbolic integration with respect to the Haar measure on the unitary groups, Bull. Pol. Acad. Sci. Tech. Sci. 65 (2017) 21 [arXiv:1109.4244].

[51] L. Pastur and V. Vasilchuk, On the moments of traces of matrices of classical groups, Commun. Math. Phys. 252 (2004) 149.

[52] J. Ben Geloun and S. Ramgoolam, Counting tensor model observables and branched covers of the 2-sphere, arXiv:1307.6490 [INSPIRE].

[53] J. Jurkiewicz and K. Zalewski, Vacuum structure of the $\mathrm{U}(N \rightarrow \infty)$ gauge theory on a two-dimensional lattice for a broad class of variant actions, Nucl. Phys. B 220 (1983) 167 [INSPIRE].

[54] E. Witten, Anti-de Sitter space and holography, Adv. Theor. Math. Phys. 2 (1998) 253 [hep-th/9802150] [INSPIRE].

[55] E. Witten, Anti-de Sitter space, thermal phase transition and confinement in gauge theories, Adv. Theor. Math. Phys. 2 (1998) 505 [hep-th/9803131] [INSPIRE]. 